\title{
State of the Art in Quorum-Based Sleep/Wakeup Scheduling MAC Protocols for Ad Hoc and Wireless Sensor Networks
}

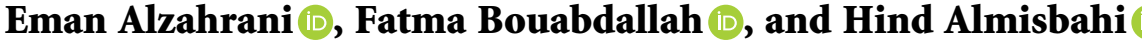 \\ Information Technology Department, Faculty of Computing and Information Technology, King Abdulaziz University, \\ Jeddah, Saudi Arabia \\ Correspondence should be addressed to Eman Alzahrani; eshalzahrani1@kau.edu.sa
}

Received 22 December 2020; Revised 24 October 2021; Accepted 11 January 2022; Published 8 February 2022

Academic Editor: Cong Pu

Copyright (C) 2022 Eman Alzahrani et al. This is an open access article distributed under the Creative Commons Attribution License, which permits unrestricted use, distribution, and reproduction in any medium, provided the original work is properly cited.

\begin{abstract}
In energy-constrained networks, designing an energy-efficient MAC protocol is crucial since the MAC protocol controls the access of the nodes to the shared wireless medium. Indeed, an efficient MAC protocol should reduce or even mitigate the consumption of the main sources of energy wastage, namely, overhearing, collision, and idle listening, and hence the network lifetime will be improved. One of the most efficient solutions is designing a MAC protocol that utilizes the quorum concept to increase energy efficiency by reducing idle listening and overhearing. Researchers have proposed several quorum-based MAC protocols to conserve energy. This paper presents a detailed overview and classification of several existing quorum-based sleep/ wakeup scheduling MAC protocols. We classify these quorum-based MAC protocols into homogeneous and heterogeneous based on their quorum system characteristics. Moreover, we further classify these protocols according to the guaranteed intersection between any two quorums into fixed and limited. Furthermore, we compare these protocols in terms of their quorum system characteristics, limitations, and performance. Finally, a guide for future research directions is presented.
\end{abstract}

\section{Introduction}

Ad hoc networks and wireless sensor networks (WSNs) are energy-constrained networks where nodes rely on batteries with limited power. Due to this energy constraint, energy conservation is an important task in these networks. The main sources of energy consumption are (i) reception, (ii) transmission, (iii) idle listening, (iv) overhearing, (v) collision, and (vi) control packet overhead $[1,2]$. The energy consumption during the transmission and reception of data is the useful energy consumption that enables nodes to accomplish their tasks. However, energy consumption due to idle listening, overhearing, collision, and control packet overhead are the main sources of energy wastage and cannot be tolerated in such energy-constrained networks. They have to be minimized to the maximum possible extent. To overcome these challenges, designing an efficient MAC protocol that is energy-efficient is crucial as the MAC protocol is in charge of coordinating the access of the nodes to the wireless medium, and hence the main sources of energy wastage can be reduced.

Many proposals have been put forward to minimize overhearing and idle listening through the use of the sleep/ wakeup scheduling [2-32]. Ideally, a node will wake up only to send or receive useful data that are destined to it, and hence idle listening, overhearing, and collision are completely avoided. That being said, the main challenge faced by these solutions is how to synchronize the sleep/wakeup schedule between a sender and its intended receiver without any extra overhead packet exchange. One of the most efficient solutions is using the quorum concept. Several quorum-based MAC protocols [7, 12-32] are proposed. The quorum-based sleep/wakeup scheduling protocols reduce nodes' wakeup time, and hence significant energy is saved. In these protocols, the quorum system is utilized to share the time slots between nodes. Indeed, the time in these protocols is split into cycles that contain fixed-size time slots. The quorum system is built from a universal set that includes 
cycle's slots numbers. A quorum system is defined as a set of non-empty subsets, and each is referred to as a quorum. Each quorum represents the node's wakeup schedule as it contains the time slots in which the node will be in the wakeup mode. A quorum will be assigned to each node. According to the quorum system, any two quorums will have an intersection. Hence, any two nodes will have at least an intersecting wakeup slot for data transmission. The rationale behind the conception of the quorum-based MAC protocols is to regulate access to the channel such that idle listening and overhearing is avoided, and thus the network lifetime will be further improved. The network lifetime is the time till nodes that are one hop away from the sink die where the network is no longer operable [30]. Quorum-based MAC protocols extend the network lifetime by reducing nodes' wakeup times, which minimizes the nodes' energy consumption resulting from idle listening and overhearing.

This survey paper is the pioneer survey dealing with all the quorum-based sleep/wakeup scheduling MAC protocols. It is true that the work in [33] deals with quorum-based MAC protocols, but only with one precise class, which is the asynchronous protocol. However, our survey is more general as we will discuss several synchronous and asynchronous quorum-based protocols. The key contribution of this paper is to survey and classify the existing quorum-based sleep/wakeup scheduling protocols. In this paper, the quorum-based MAC protocols will be classified according to their quorum system characteristics into homogeneous and heterogeneous. Moreover, these protocols are also classified according to the guaranteed intersection between any two quorum sets into fixed and limited. This classification will help researchers in choosing the direction of research in the coming days.

In this paper, Table 1 lists the abbreviations used throughout the paper. The rest of the paper is organized as follows. Section 2 introduces the quorum concepts. In Section 3, we survey and classify several quorum-based sleep/wakeup scheduling protocols. In Section 4, we discuss the possible direction for future research. Finally, we conclude the paper in Section 5.

\section{Quorum Concept}

A-quorum concept has been widely applied in mobile ad hoc networks for location management $[34,35]$, in WSNs for data aggregation [36] and information dissemination [37], in distributed systems for replica control problems [38, 39] and mutual exclusion [40], and for multichannel MAC protocol design in underwater sensor networks [41, 42]. The following is a definition of the quorum system.

Definition 1. A-quorum system $Q$ under a universal set $U=$ $\{0, \ldots, n-1\}$ is a collection of non-empty subsets of $U$, and each subset is called a quorum, which satisfies the following intersection property:

$$
\forall G, H \in Q ; G \cap H \neq \varnothing .
$$

TABLE 1: List of abbreviations.

\begin{tabular}{lc}
\hline Abbreviation & Meaning \\
\hline WSNs & Wireless sensor networks \\
IoT & Internet of Things \\
PSM & Power-saving mode \\
BIs & Beacon intervals \\
HBI & Half-awake beacon interval \\
SBI & Sleep beacon interval \\
BW & Beacon window \\
DW & Data window \\
SIFS & Short Interframe Space \\
LAPS & Location-aware power saving \\
QMMAC & Quorum-based multichannel MAC \\
S-grid & Stepped grid \\
AQEC & Adaptive quorum-based energy conserving \\
HQPS & Hyper-quorum system-based power saving \\
AS-grid & Adaptive S-grid \\
TLS & Traffic load-based scheduling \\
HMAC & Homogeneous MAC \\
AQPS & Adaptive quorum-based power saving \\
OFAA & Optimal fully adaptive and asynchronous \\
ACQ & Asymmetric cyclic quorum \\
GQS-pair & Grid quorum system pair \\
CQS-pair & Cyclic quorum system pair \\
AAA & Asynchronous, adaptive, and asymmetric \\
AMQ & Asymmetric majority quorum \\
QMAC & Quorum-based MAC \\
QTSAC & Element shift quorum \\
ESQ & Multiclass quorum system \\
MCQS &
\end{tabular}

For example, $Q=\{\{1,3\},\{1,2\},\{2,3\}\}$ is a quorum system with three non-empty subsets $\{1,3\},\{1,2\}$, and $\{2,3\}$ under the universal set $U=\{1,2,3\}$. There are several kinds of quorum systems, including torus [43], cyclic [44], grid [45], and others [46-48].

\section{Quorum-Based Power-Saving Protocols}

Many sleep/wakeup scheduling protocols [12, 14-26] are designed on top of IEEE 802.11 power-saving mode (PSM) [49]. PSM allows nodes to enter the sleep mode if there is no data transmission. In this power-saving protocol, the time is partitioned into beacon intervals (BIs), and each is divided into ATIM window and data period. At the start of each ATIM window, a beacon frame is sent based on contention to synchronize nodes. When a beacon frame is heard, the node synchronizes its clock and cancels its beacon frame transmission. A node requires to be awake throughout the ATIM window. The node wakes up during the entire BI for data reception if an ATIM frame is received. If no ATIM frame is received when the node reaches the end of the ATIM window, the node goes back to the sleep mode. Figure 1 shows an example. At the ATIM window in the first $\mathrm{BI}$, if no ATIM frame is received, nodes A and B return to the sleep mode after ATIM window. In the second BI, A wants to send a packet to B. A sends ATIM frame at the ATIM window. B replies with an ATIM ACK. Then, A will try to transmit the data packet after the ATIM window. 


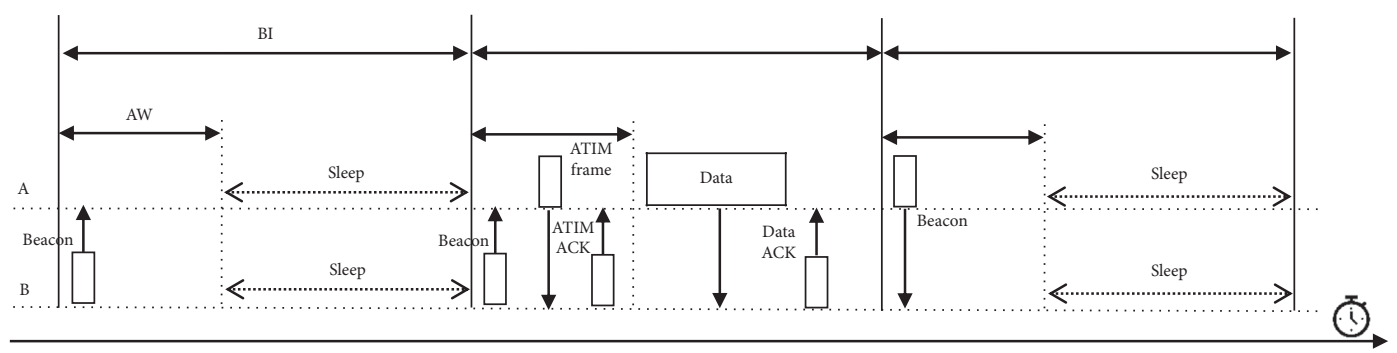

Figure 1: IEEE 802.11 PS mode.

It is true that PSM is allowing a node to save more energy by switching to sleep mode if no packet transmission is required. However, a node requires to be awake for a fixed time for every BI (ATIM window) even if the traffic load is light. Moreover, in multihop networks, this protocol suffers from costly clock synchronization problem. Therefore, many researchers proposed power-saving protocols based on the quorum concept to overcome these problems. Quorumbased MAC protocols are conceived to save further energy, and hence the network lifetime will be increased. In these quorum-based protocols, the time is partitioned into cycles of fixed-size time slots. In each cycle, slots are either quorum slots or non-quorum slots. The node wakes up at the quorum slot to receive and send data and goes to sleep mode during the non-quorum slot to save energy. A-quorum system is built from a universal set of slots numbers $U$. In the quorum system, a quorum is a subset of $U$ and represents the nodes' wakeup time slots. For example, in Figure 2, the quorum $Q_{A}=\{0,1,4,7\}$ is assigned to node $\mathrm{A}$, which means A wakes up at time slots $0,1,4$, and 7 . Any two neighbors must be assigned two quorum sets with a number of intersecting quorum slots in order to allow communication. For instance, as depicted in Figure 2, nodes A and B are assigned two different quorum sets. A is assigned quorum with elements $0,1,4$, and $7 . \mathrm{B}$ is assigned quorum with elements $0,3,4$, and 6 . A and B can communicate at the intersecting wakeup slots 0 and 4 where $Q_{A} \cap Q_{B}=\{0,4\}$.

To apply this concept, many questions need to be answered. How to build these quorums such that mutual intersections are guaranteed between any two quorums? How many intersections? How to assign quorums to nodes? What is the suitable cycle length? Should it be the same for all nodes or different? Many literature works have been done to address these questions [7, 12-31]. Most importantly, they are different in terms of building the quorum sets to guarantee their application requirement in terms of end-toend delay, throughput, and traffic load (burst traffic or variant traffic). Moreover, these protocols differ in their quorum system characteristics such as the quorum size, the expected number of intersections, and the activity ratio (the number of quorum slots to the cycle length).

The quorum-based sleep/wakeup scheduling protocols can be classified according to their quorum system characteristics as homogeneous and heterogeneous. The protocol is called homogeneous if nodes are assigned quorums with the same characteristics. On the other hand, if the protocol allows nodes to have quorums with different characteristics,

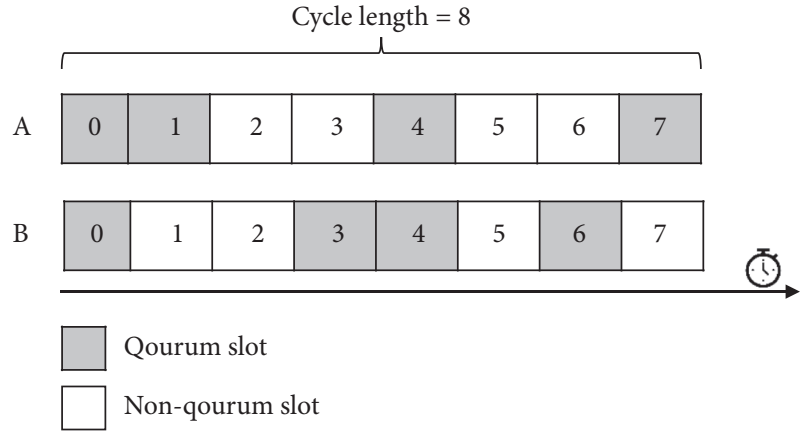

FIGURE 2: Example of intersecting quorums.

this protocol is referred to as a heterogeneous protocol. The heterogeneous protocols can be classified into adaptive, asymmetric, and adaptive asymmetric. In the adaptive protocols, nodes can dynamically adapt their quorums whenever their situation changes. On the other hand, if the protocol allows nodes to have quorums from two or more different quorum systems, generally two, this protocol is referred to as asymmetric protocol. In this asymmetric protocol, the intersection between nodes may not be guaranteed. Differently, the quorum-based sleep/wakeup scheduling protocols can be classified according to the guaranteed intersection between any two quorum sets into fixed or limited. The fixed ones either allow exactly the same number of intersections between any two quorums or determine a specific number of intersections based on nodes' chosen parameters. For example, LAPS [12] protocol allows one intersection between any two nodes. In a different way, in QueenMAC [27], two nodes $n_{1}$ and $n_{2}$ will choose $k_{1}$ and $k_{2}$ values, respectively, and the intersection between these two is equal to $k_{1} \times k_{2}$. Note that $k_{i}$ is a protocol parameter that depends on node $i$ traffic load. In the limited intersection case, the number of intersections is variable but bounded. For example, the number of intersections in grid quorum-based protocol is greater than 2 and less than $n-\sqrt{n}+1$, where $n$ is the quorum system size. Accordingly, the existing quorum-based sleep/wakeup scheduling protocols can be placed within one of the following classifications: (i) homogeneous with fixed intersection size, (ii) homogeneous with limited intersection size, (iii) adaptive heterogeneous with limited intersection size, (iv) asymmetric heterogeneous with limited intersection size, (v) adaptive asymmetric heterogeneous with fixed intersection size, and (vi) adaptive asymmetric heterogeneous with 
limited intersection size. The classification is illustrated in Figure 3.

\subsection{Homogeneous Protocols with Fixed Intersection Size.} An IEEE 802.11PSM-based protocol named location-aware power-saving protocol (LAPS) was proposed in [12]. This protocol is designed for multihop mobile ad hoc networks. In this protocol, the quorum system is built such that nonneighbor nodes will have zero intersecting wakeup slots. Moreover, this protocol takes into account the hidden terminal problem. LASP assumes that (1) nodes are uniformly distributed, have the same transmission distance, and are time-synchronized, (2) the area is partitioned into groups (each group is further divided into seven equal-size hexagon cells), and (3) each node knows the location of the central cell and its location. Figure 4 shows a network topology example. $C_{i}^{k}$ represents the cell $i$ in group $G_{k}$ where $0 \leq i \leq 6,0 \leq k \leq m$, and $m$ is the total number of groups.

LAPS proposed a new quorum system to satisfy two constraints. The first constraint is that only nodes in neighbor cells can communicate. This constraint is achieved by assigning common quorum slots between neighbor nodes and avoiding the assignment of quorum intervals between the non-neighbor ones. The second constraint is that the two-hop neighbor nodes should be assigned different quorum slots to avoid the hidden terminal problem [50]. LAPS quorum system construction consists of two procedures: intragroup scheduling and intergroup scheduling, described later. According to LAPS, each group contains seven cells, and a quorum set from the quorum system will be assigned to each cell. Thus, the LAPS quorum system contains seven quorum sets. Each cell has six neighbor cells; therefore, each quorum set has six slots, one wakeup slot for each neighbor. Initially, the quorum sets will be constructed from the universal set $U=\{0,1, \ldots, 35\}$. The first six sets will be assigned an element form $U$, and the last set will be kept without any assignment. Figure 5 shows the initial quorum set design.

The intragroup scheduling procedure is designed to assign a common quorum slot between any two neighbors in the same group. Figure 6 illustrates the neighboring relationship in the group. At this procedure, there are two steps. Suppose $A[i, q]$ represents element $i$ in quorum set $q$. At the first step, the value of element $A[q-1, q]$ will be copied to $A[q-1,6]$ and then replaced by $A[q-1,0]$, for each $q$ where $1 \leq q \leq 6$. Hence, each cell will have a common slot with the central cell $C_{0}^{k}$. The result of the first step is shown in Figure 7 (a). At the second step, a common quorum slot will be assigned to the remaining neighboring relationship, shown in red arrows in Figure 6 . To do this, element $A[i, q]$ is replaced by $A[i, j]$ for $0 \leq i \leq 5$ where $q= \begin{cases}i+5, & \text { if } i \leq 1 \\ i-1, & \text { if } i>1\end{cases}$ and $j=\left\{\begin{array}{ll}i+6, & \text { if } i=0 \\ i, & \text { if } i>0\end{array}\right.$. The result of the second step is shown in Figure $7(\mathrm{~b})$.

In the second procedure, the intergroup scheduling procedure, a common quorum slot will be assigned to any two neighbors belonging to different groups while avoiding the hidden terminal problem. As illustrated in Figure 8(a), group $G_{k}$ has six neighboring groups $G_{K+1}, G_{k-1}, G_{a}$, $G_{a+1}, G_{b}, G_{b+1}$. In this figure, 18 arrows represent the neighboring relationship in these groups. Indeed, there are duplicated arrows. For example, as shown in Figure 8(b), the quorum slots between $C_{3}^{a}$ and $C_{1}^{k}$ can be reused for $C_{3}^{k}$ and $C_{1}^{b+1}$. Likewise, the common slots between $C_{4}^{a}$ and $C_{1}^{k}$ and $C_{5}^{a+1}$ and $C_{1}^{k}$ can be reused for $C_{4}^{k}$ and $C_{1}^{b+1}$ and $C_{5}^{k}$ and $C_{1}^{b}$, respectively. Accordingly, as illustrated in Figure 8(c), for intergroup scheduling, nine quorum slots are needed. As illustrated in Figure 9, for $C_{1}^{k}$, element $A[1,3]$ will be replaced by $A[3,1]$. Elements $A[2,4]$ and $A[3,5]$ will be replaced by $A[4,1]$ and $A[5,1]$. In the same way, for $C_{2}^{k}$, elements $A[0,4], A[2,5]$, and $A[4,6]$ will be replaced by $A[0,2], A[4,2]$, and $A[5,2]$, respectively. For $C_{3}^{k}$, elements $A[1,5]$ and $A[3,6]$ will be replaced by $A[0,3]$ and $A[5,3]$. For $C_{6}^{k}$, element $A[2,6]$ will be replaced by $A[1,4]$. The result of the intragroup scheduling procedure is shown in Figure 10(a). After subtracting the number of the unused slots, the universal set size will be $21, U=\{0,1, \ldots, 20\}$. After rearranging the quorum slots, the resultant quorum system is shown in Figure 10(b).

Finally, a cell identification scheme has been proposed. In this scheme, depending on the node location and the central cell location $C_{0}^{1}$, each node can define its group and cell IDs. Thus, each node can determine its assigned quorum.

LAPS protocol succeeds to prolong the network lifetime compared to PSM. Moreover, it reduces the collision due to hidden terminal problem. Furthermore, LAPS performs better in terms of end-to-end delay, packet loss ratio, and energy efficiency especially in high-density networks compared to PSM and AQBC [16]. However, more than one node may exist in the same cell which may introduce a collision, and hence the network performance may be degraded.

\subsection{Homogeneous Protocols with Limited Intersection Size}

\subsubsection{Quorum-Based Multichannel MAC Protocol} (QMMAC) [13]. QMMAC is a MAC protocol that uses the quorum concept as well as the multichannel communication feature to highly increase throughput while saving energy. This protocol is proposed for corona-based networks where the sink is located at the center and nodes are arranged into coronas with equal width based on their hop counts from the sink. Figure 11 illustrates a five-corona network. QMMAC aims at allowing forwarders nodes to wake up at the same time while separating these wakeup forwarders utilizing the multichannel feature. This protocol is composed of two steps: (i) quorum-based sleep/wakeup scheduling and (ii) quorum-based data channel assignment for multichannel communications.

First, the quorum-based sleep/wakeup scheduling is built to avoid overhearing and idle listening and reduce the endto-end delay. In this sleep/wakeup scheduling, any node and its upstream forwarders will wake up during the same slots, whereas remaining neighbors will wake up at entirely different slots. To do so, the line quorum system was proposed where every quorum is composed only of one line from a 


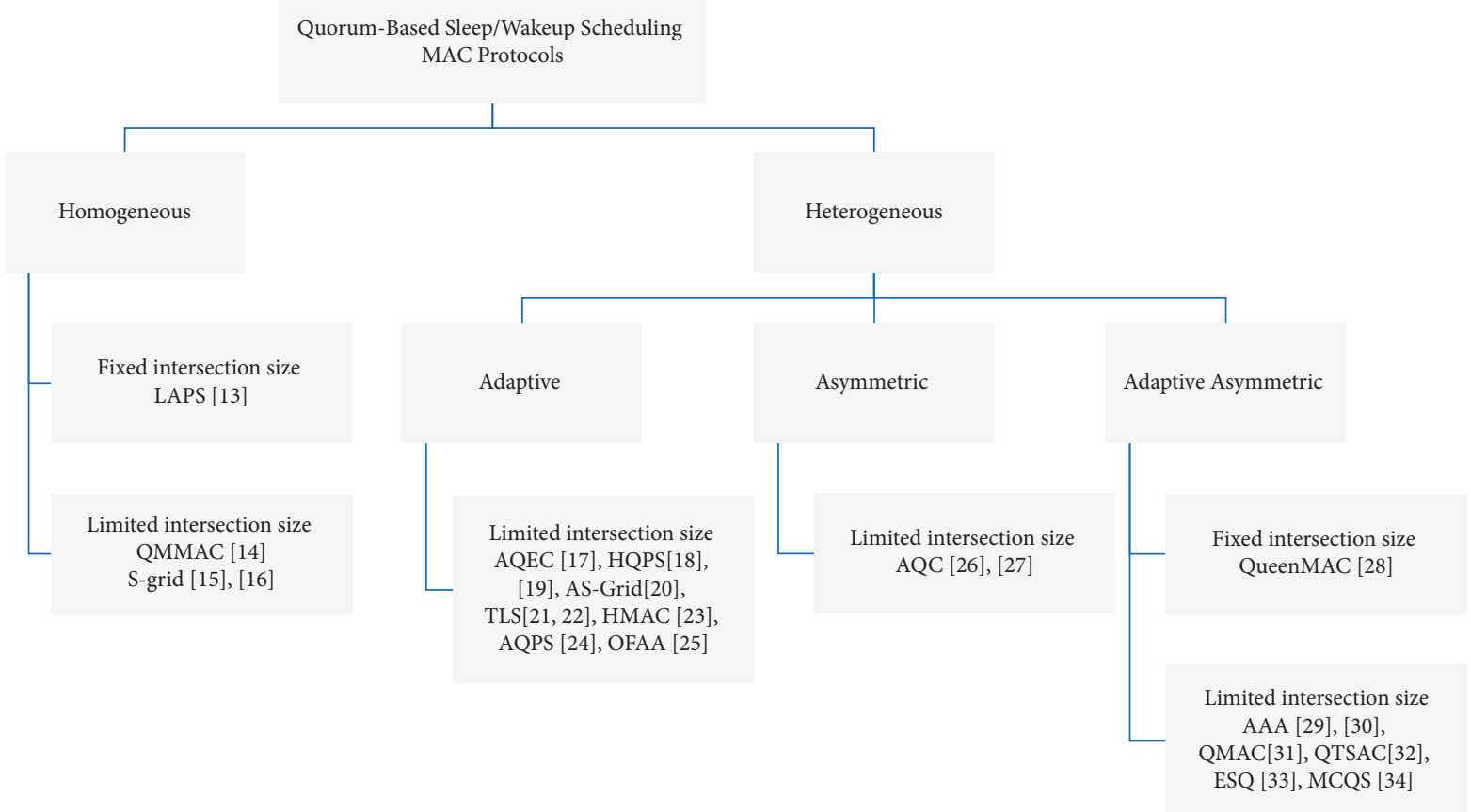

Figure 3: The classification of quorum-based sleep/wakeup scheduling protocols.

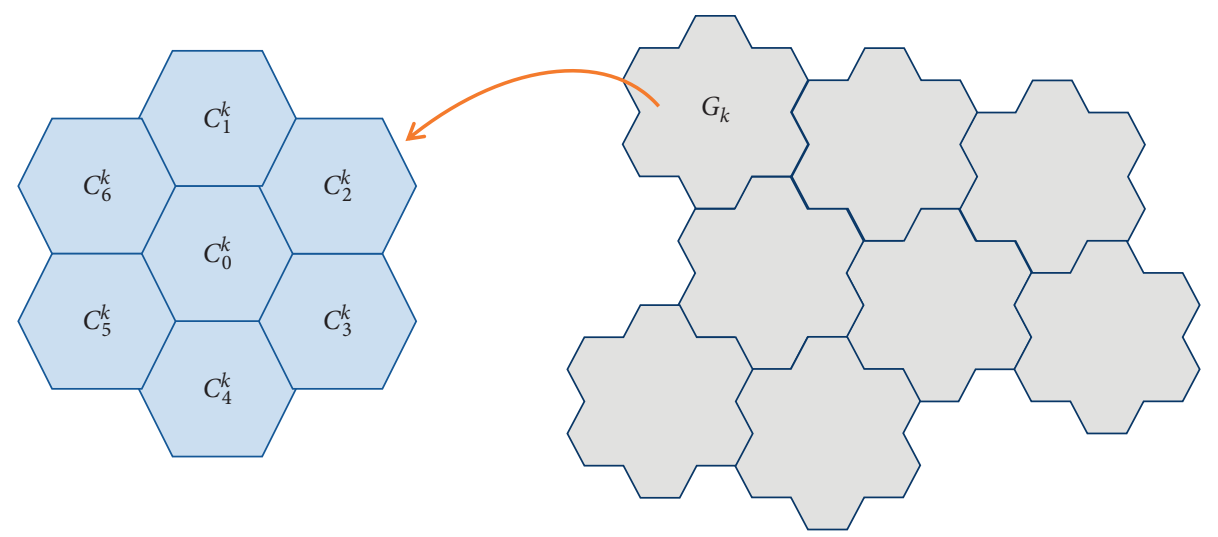

FIGURE 4: An example of LAPS network topology.

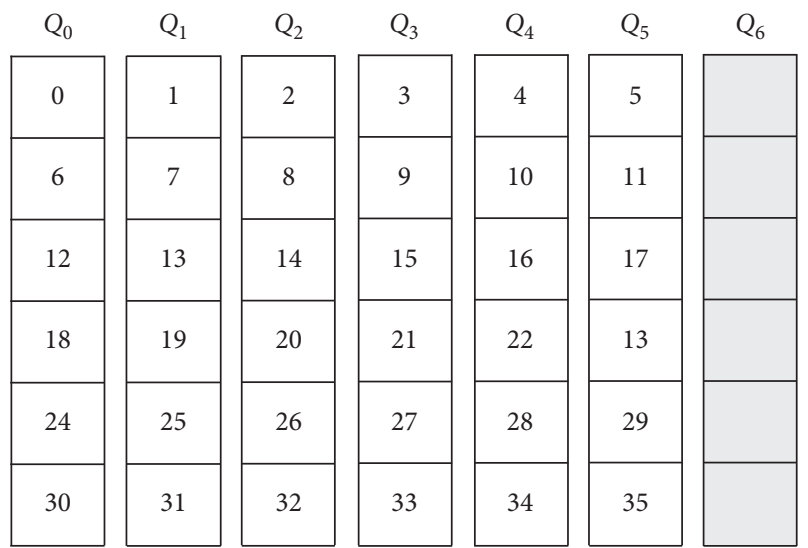

FIgURE 5: The initial quorum set design.

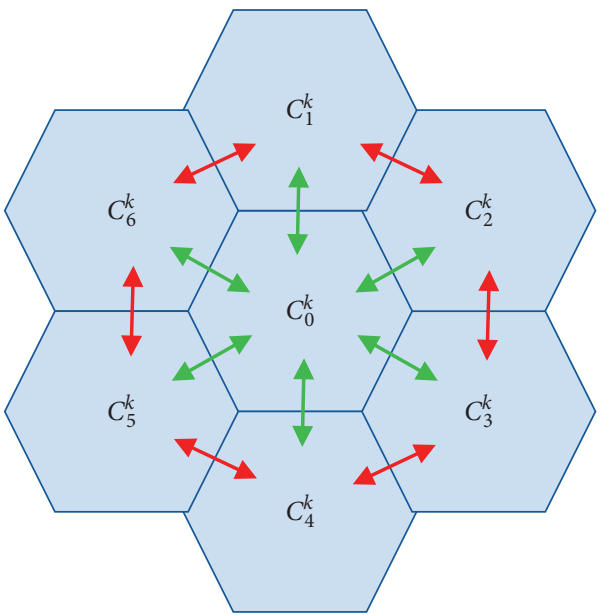

FIGURE 6: The neighboring relationship in the group. 


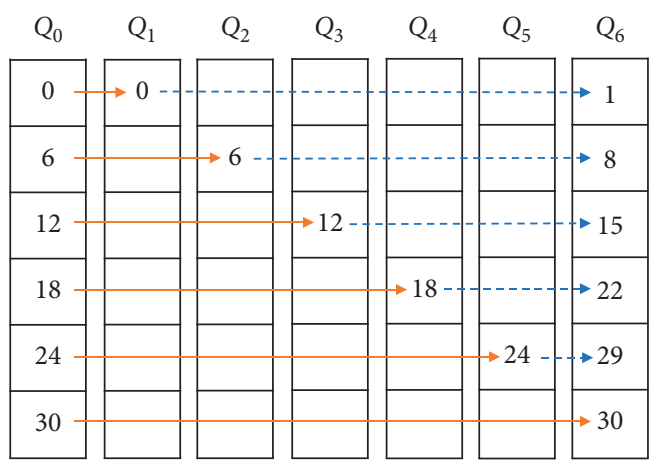

(a)
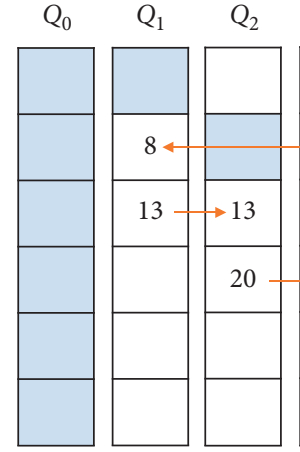

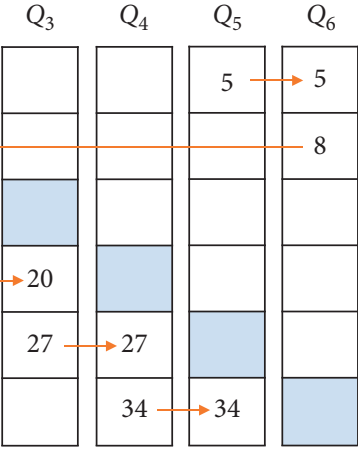

(b)

Figure 7: Intragroup scheduling steps. (a) Step 1. (b) Step 2.

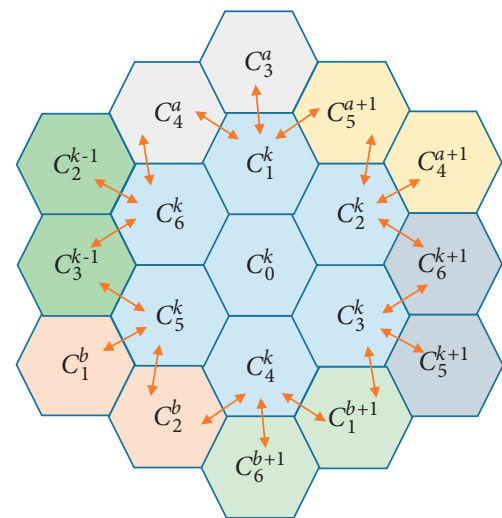

(a)

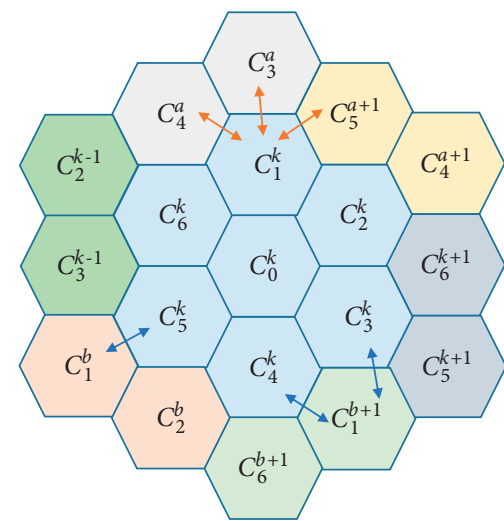

(b)

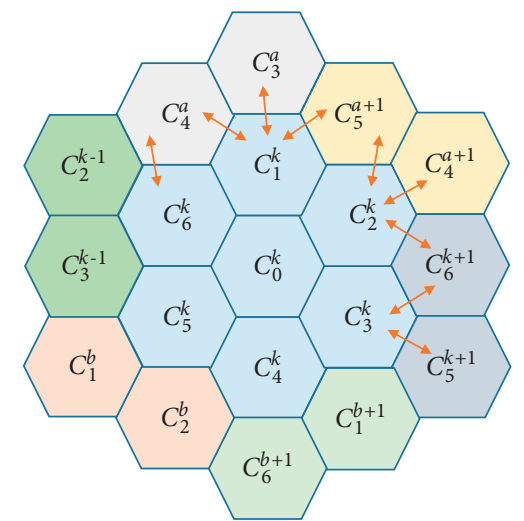

(c)

FIGURE 8: Intergroup neighboring relationship.

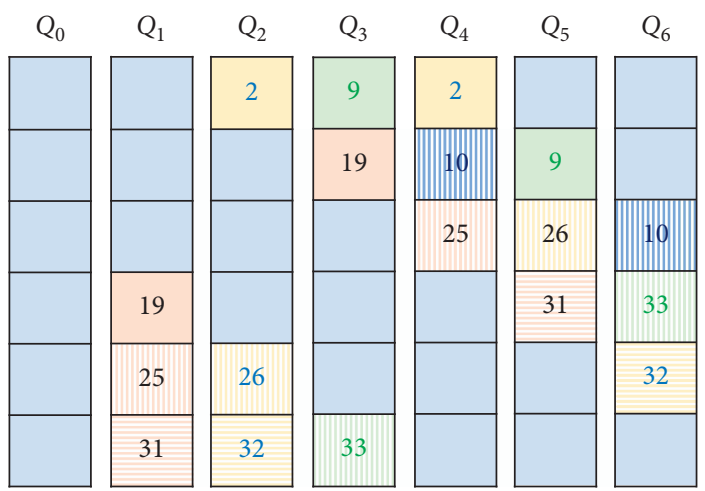

FIGURE 9: Intergroup scheduling interval replacement.

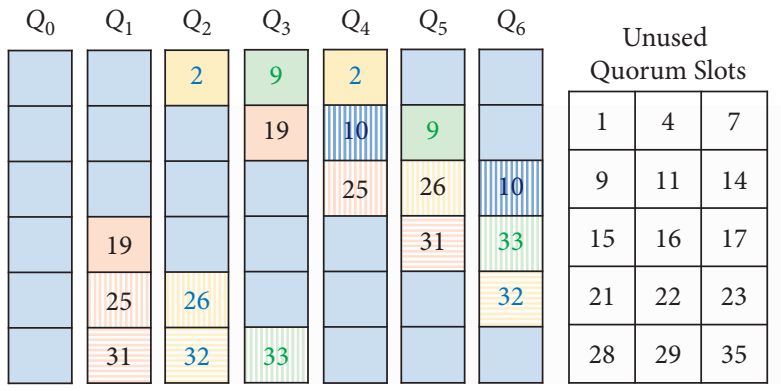

(a)

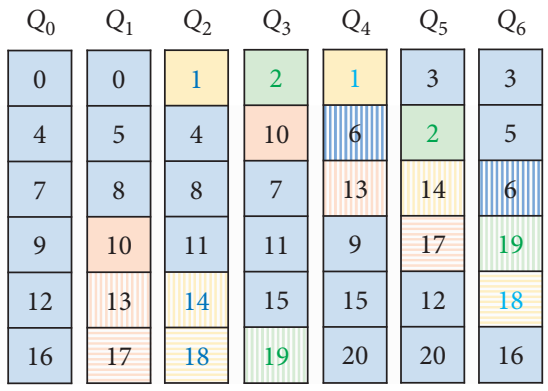

(b)

FIGURE 10: The resultant quorum system after the intergroup scheduling. 


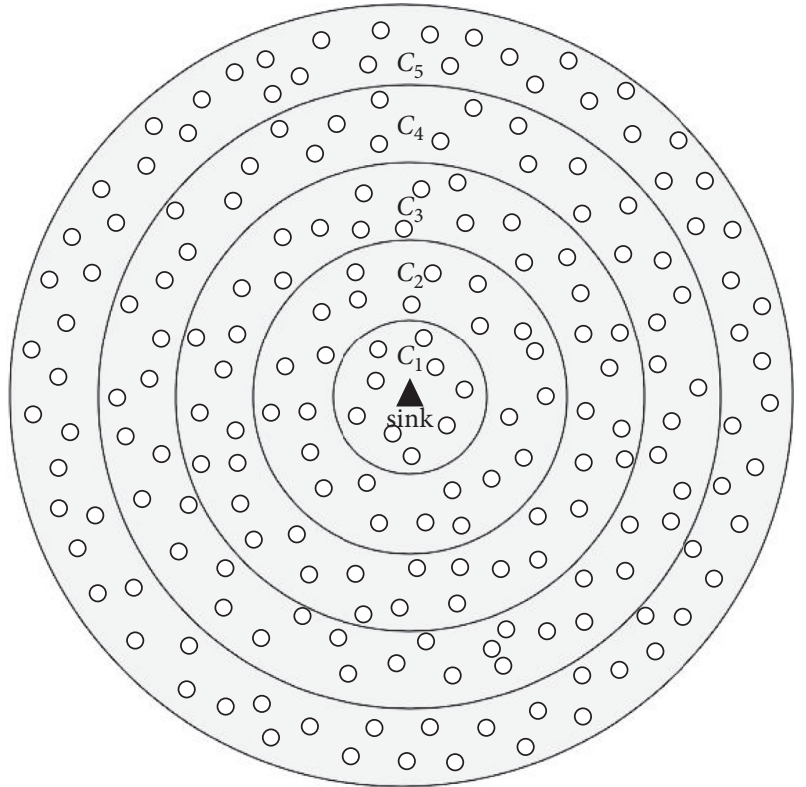

FIgURE 11: QMMAC network topology.

$n \times s$ grid. Initially, a node in the last corona, $C_{5}$ in Figure 11, is assigned a quorum as its wakeup schedule. This quorum system guarantees that nodes in the same corona that are within a two-hop neighborhood have zero intersecting quorum slots by assigning them different quorums (different lines). Then, to reduce end-to-end delay, the node's upstream forwarders are assigned exactly the same quorum. As illustrated in Figure 12, A and all its next-hop forwarders (shown in the blue sector) are assigned line $\mathrm{L} 1$, whereas $\mathrm{B}$, a neighbor of $\mathrm{A}$, is assigned line L2. The intersecting nodes will end up with a longer active ratio since they wake up first at slots $0,1,2$, and 3 with node $\mathrm{A}$ and then wake up at slots $4,5,6$, and 7 with $\mathrm{B}$.

In order to estimate the grid size, $n$, the number of lines, is estimated so that nodes within two-hop neighborhood in the last corona are assigned different lines to mitigate collision and hence reduce energy waste. Consequently, $n$ is the number of nodes on average in a given two-hop neighborhood and simply $n$ is expressed as described in [13] as follows:

$$
n=d \times A,
$$

where $d$ is the network density and $A$ is the area representing a two-hop neighborhood. Next, the number of slots $s$ [13] is determined so that there are enough slots for every node to transmit its data. Accordingly, $s$ is derived as follows:

$$
s=N_{\text {avg }} \times H_{\text {avg }},
$$

where $N_{\text {avg }}$ is the average number of nodes waking up on the line and $H_{\text {avg }}$ is the average number of hops.

Once the grid size is defined, QMMAC assigns the last corona nodes different lines in such a way that two-hop neighbor nodes will be allocated a unique line $l$ from $n \times s$ grid. As depicted in Figure 13, the circular area is divided into equalwidth sectors such that in each sector, there is one node. The number of the sector in which node $u$ is located is defined as $q$.
Therefore, $q$ is a unique number that represents node $u$, and nodes in a given area $A$ can be allocated a unique line $l$ [13] between 0 and $n-1$ using the modulus operator as follows:

$$
l=q \bmod n .
$$

Second, a quorum-based channel assignment is used for multichannel communications in order to share the available channels without neither conflict nor extra packet exchange to negotiate channel availability, and hence collisions between conflicting neighbor nodes are avoided and the end-to-end delay is decreased. This protocol is built on top of IEEE 802.15.4 which has sixteen non-overlapping channels. QMMAC uses sixteen non-overlapping channels, one common control channel, and fifteen data channels. The control channel's time is split into $n \times s$ slots. Every node in QMMAC has a unique channel with each one of its upstream forwarders for data communication. It utilizes the singleton-intersection quorum system [40]. A quorum in this quorum system is a subset of data channels to be used by the node. Furthermore, any two quorums intersect in a different singleton. The singleton-intersecting quorum system is expressed as follows.

Theorem 1 (see [40]). Given $t$, the system $C=S_{1}, \ldots, S_{t}$, where

$$
\left\{\begin{array}{l}
\forall 1<j \leq M ; \operatorname{card}\left(S_{j}\right)=M-1, S_{1}=\{1,2, \ldots, M-1\} \\
\text { and } S_{j}=\left\{\left(S_{1}\right)_{j-1}, \ldots,\left(S_{j-1}\right)_{j-1},\left(S_{j-1}\right)_{M-1}\right. \\
\left.+1, \ldots,\left(S_{j-1}\right)_{M-1}+(M-j)\right\} \\
\left(S_{p}\right)_{q} \text { refer to the } q \text { th element of } S_{p}
\end{array}\right.
$$

is a unique singleton-intersecting quorum system under $U=$ $\{1, \ldots, M\}$ where $M$ represents the different elements in the quorum system and is equal to $t(t-1) / 2$, where $t$ is equal to the number of nodes sharing the quorum system.

QMMAC quorum system is built as a unique singletonintersecting quorum system where $M=15$ since there are 15 different data channels. Thus, every node has five upstream forwarders at most. Accordingly, the QMMAC quorum system has six sets each containing five elements as follows: $S_{1}=\{1,2,3,4,5\}, S_{2}=\{1,6,7,8,9\}, S_{3}=\{2,6,10,11,12\}$, $S_{4}=\{3,7,10,13,14\}, \quad S_{5}=\{4,8,11,13,15\}, S_{6}=\{5,9,12$, $14,15\}$.

QMMAC slot and channel assignments provide collision-free communication by assigning wakeup nodes in a given neighborhood, a unique channel, and a unique slot. However, when two neighbors are assigned the same channel set, these two neighbor nodes may experience a collision if and only if they miss each other's CTS. A node may miss its neighbors' CTS if it is busy communicating at data channel while they are sending CTSs at the common control channel.

QMMAC protocol succeeds to conserve more energy, improve the network lifetime, and reduce end-to-end delay compared to grid quorum systems. Moreover, QMMAC 


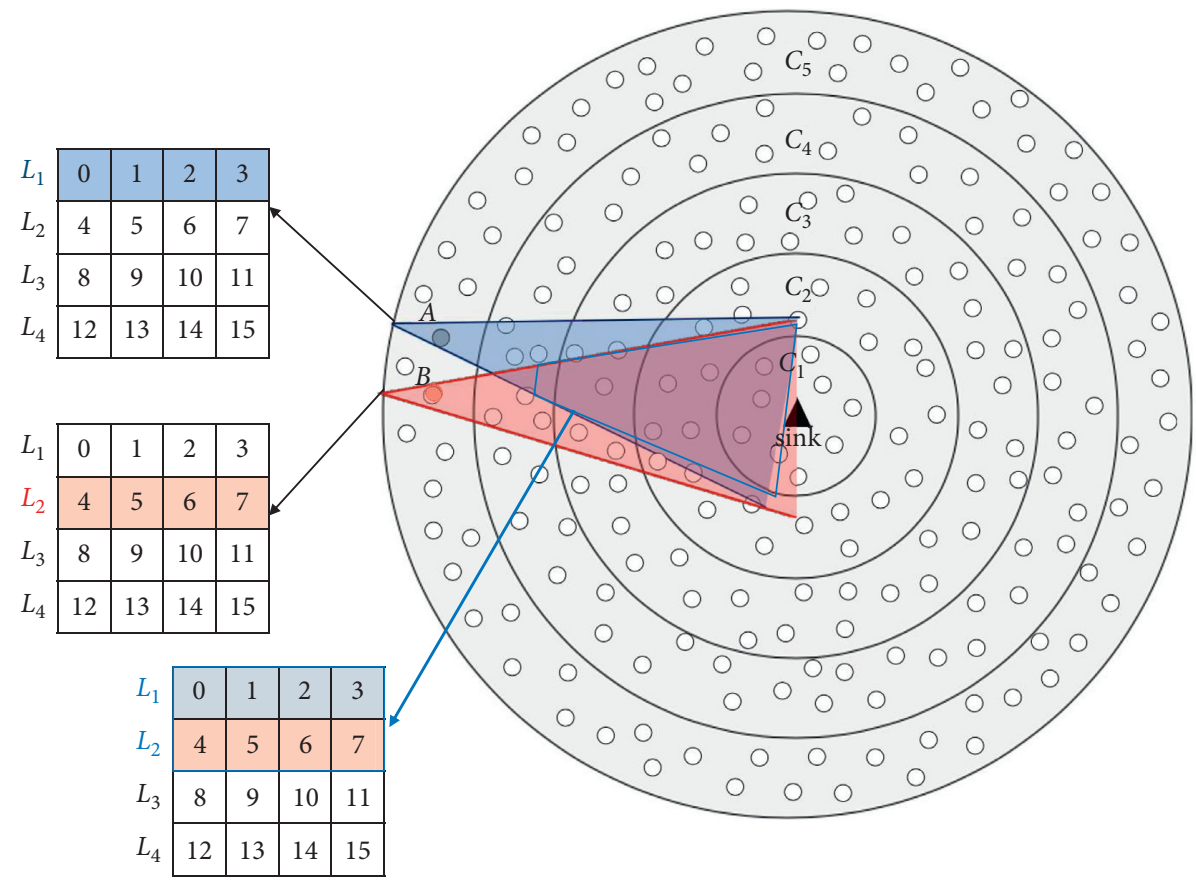

FIGURE 12: Intersecting nodes.

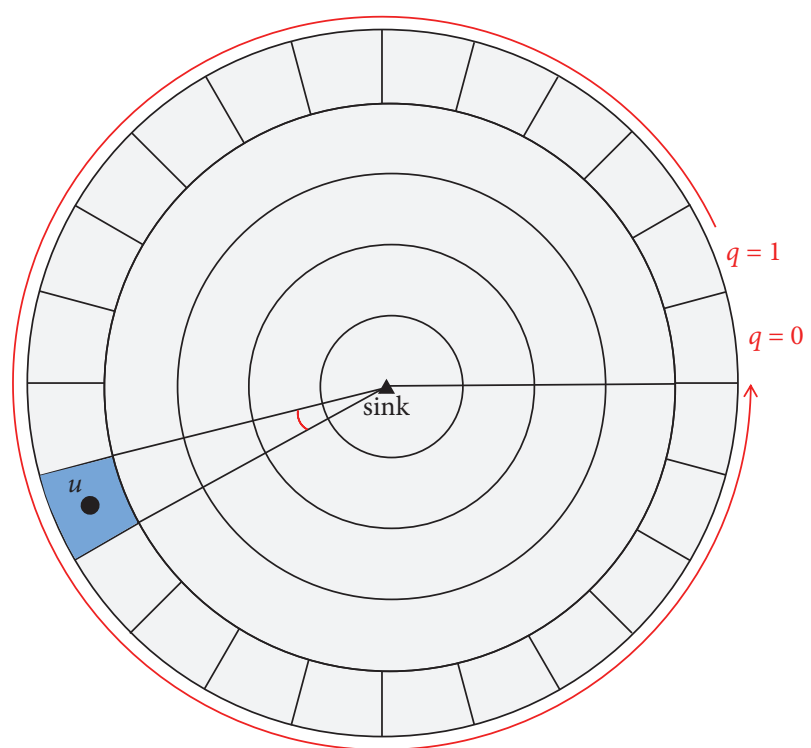

FIgURE 13: Partitioning the last corona into equal-width sectors.

used the multichannel communication feature to extremely increase the throughput and further save energy. However, it may suffer from collision.

3.2.2. Stepped Grid (S-Grid) Quorum System [14]. In [14], a non-adaptive symmetric quorum-based protocol was proposed. In this paper, a new quorum system named stepped grid (S-grid) is proposed. The S-grid is a quorum system where each node selects its wakeup slots from a $t \times w$ grid, where $t$ may be equal or not to $w$. Each node selects $(w)+$ $(t-1)$ slots as follows:
(1) All slots in row $i$ where $0 \leq i \leq t-1$.

(2) Slots in column 0 , starting from slot 0 to the first slot in row $i$.

(3) Slots in column $w-1$, starting from the last slot in row $i$ to the last slot $t \times w-1$.

According to S-grid, nodes can randomly choose from $t$ different quorum sets. For example, as shown in Figure 14, if $t=3$ and $w=4$, three possible quorum sets can be selected by nodes. This quorum system is proposed as an alternative one to the grid quorum system with better neighbor sensibility. The neighbor sensibility is the worst-case delay for a node to discover a new node in its neighborhood [47]. This protocol provides better neighbor sensibility by increasing the number of intersections with the increase of the system size. As in the grid quorum system, the S-grid guarantees that two nodes with different quorum sets have at least two intersecting wakeup slots. However, all nodes have the same grid size and the same activity ratio regardless of their situation. Moreover, this protocol is an asynchronous protocol; thus, nodes have to remain awake during the quorum slot to ensure slot intersection. Also, they are vulnerable to control packet collisions when neighbor nodes start communication simultaneously since they follow the IEEE 802.11 PSM BI structure.

3.2.3. Continuous Quorum-Based Multicast Power-Saving Protocol [15]. Two quorum systems C-Grid and C-Torus were proposed in [15] to allow nodes to transmit burst unicast traffic. Furthermore, to allow nodes to transmit burst multicast traffic, CRT $C(m)$-arbiter and uniform $C(m)$ arbiter quorum systems are proposed such that the nodes have continuous intersecting slots between $m$ quorums. The 

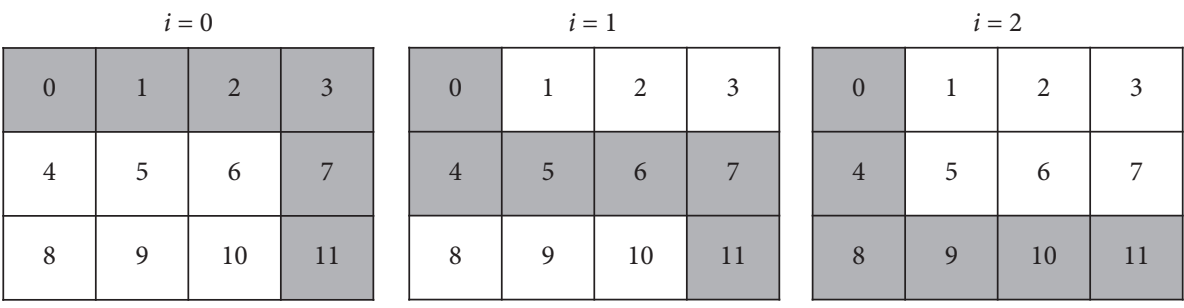

Figure 14: Possible quorum sets on S-grid $(3 \times 4)$.

C-Grid is a grid quorum system where each node selects $s$ rows and $d$ columns from $\sqrt{n} \times \sqrt{n}$ grid, where $2 \leq s, d \leq \sqrt{n}$. C-Grid guarantees that in each selected column, there are at least $d$ continuous intersections. As shown in Figure 15, A and $B$ select two rows and two columns as their wakeup schedules. Accordingly, $Q_{A}=\{4,5,10,11,16,17,22$, $23,24,25,26,27,28,29,30,31,32,33,34,35\}$ and $Q_{B}=\{0,1$, $2,3,4,5,6,7,8,9,10,11,12,13,18,19,24,25,30,31\}$ where the intersecting wakeup slots between them are $Q_{A} \cap Q_{B}=\{4,5,10,11,24,25,30,31\}$. Note the continuity between successor intersection slots which will allow burst traffic transmission. This paper proposes another quorum system C-Torus for unicast transmission. Each node selects $s$ columns along with $w / 2-s+2$ continuous numbers of slots from $t \times w$ grid. C-Torus guarantees at least two continuous intersections between any two nodes. As depicted in Figure 16, two nodes, node A and node B, select two columns and four continuous numbers from $3 \times 8$ grid as their wakeup schedules. Accordingly, 2 and 3 are the continuous intersections between them.

To enable unicast transmission, a continuous asynchronous power-saving protocol is proposed. In this protocol, each quorum slot is divided into a beacon window, a MTIM window, and data period. First, a beacon message is sent to indicate that the node is awake. Then, during the MTIM window, the node sends a MTIM packet to ask its receiver to wake up a number of continuous slots. If the MTIM packet is received, the receiver sends back an ACK to inform the sender about the number of continuous slots it can awake based on its schedule. After receiving the ACK, the node transmits burst traffic throughout the continuous slots. At the end of the continuous slots, the receiver transmits an ACK. As shown in Figure 17, node A asks for three upcoming continuous quorum slots. Node B sends an acknowledgment for two upcoming continuous quorum slots. A will transmit its burst messages during the next two continuous quorum slots without sending control packets again. By the end of the second quorum slots, node B sends data ACK after receiving the last message.

For transmitting burst multicast traffic, a continuous asynchronous multicast power-saving protocol is proposed. This protocol allows continuous multicast transmission by adopting either CRT $C(m)$-arbiter or uniform $C(m)$-arbiter quorum systems. These quorum systems guarantee at least two continuous intersections between $m$ nodes. This protocol is proposed to outperform the busy waiting protocol problem where the sender wakes up its receivers one by one and waits until all of them wake up. An example of a busy waiting protocol is shown in Figure 18(a). Differently, in this protocol, if a node wants to send burst messages to multiple receivers, it will select a quorum system for its $m$ receivers. Then, when one of its receivers wakes up, it sends a quorum from the selected quorum system to the wakeup receiver. Receivers will change their wakeup schedules according to the received quorums. Thus, receivers can go to sleep in some beacon intervals while waiting for burst multicast messages. After notifying $m$ receivers about their quorums, the node waits for all its receivers to wake up at the same time in order to send the burst messages. Nodes return their schedules to the original after burst messages are received. Figure 18(b) shows an example of a continuous asynchronous multicast power-saving protocol.

Collisions may occur during transmission of the control packets (beacon and MTIM). Using MTIM ACK, the collision of the data packets is avoided since neighbor nodes will be aware of how many continuous slots the channel is busy with. Moreover, the MTIM ACK avoids the hidden terminal problem. However, exposed terminal problem may occur. Figure 19 demonstrates the exposed terminal problem. As depicted in Figure 19, A sends a packet to B, while C wants to send a packet to D. However, C is aware of A's transmission, and it unnecessarily postpones its transmission until the channel is idle.

This protocol reduces the end-to-end delay by $30 \%$ and increases the throughput and energy conservation by $40 \%$ compared to the non-continuous quorum-based unicast protocols (grid and tours). Moreover, it degrades the end-toend delay by $35 \%$ and increases the throughput and energy conservation by $20 \%$ compared to the non-continuous quorum-based multicast protocols. In conclusion, the continuous power-saving protocol is suitable for burst traffic; however, it is not suitable for varied traffic loads.

\subsection{Adaptive Protocols with Limited Intersection Size}

3.3.1. Adaptive Quorum-Based Energy-Conserving (AQEC) Protocol [16]. AQEC is a quorum-based MAC protocol that uses the grid quorum system to minimize nodes' idle listening time based on their traffic load. Each node chooses a row and a column from $n \times n$ grid. Then, it can adjust its grid size based on its traffic load. In AQEC, a node chooses one of the four grid sizes according to three traffic load thresholds, as described in [16]: 


\begin{tabular}{|c|c|c|c|c|c|}
\hline 0 & 1 & 2 & 3 & & \\
\hline 6 & 7 & 8 & 9 & & \\
\hline 12 & 13 & 14 & 15 & 16 & 17 \\
\hline 18 & 19 & 20 & 21 & 22 & 23 \\
\hline & $y$ & 26 & 27 & 28 & 29 \\
\hline 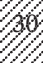 & 31 & 32 & 33 & 34 & 35 \\
\hline
\end{tabular}
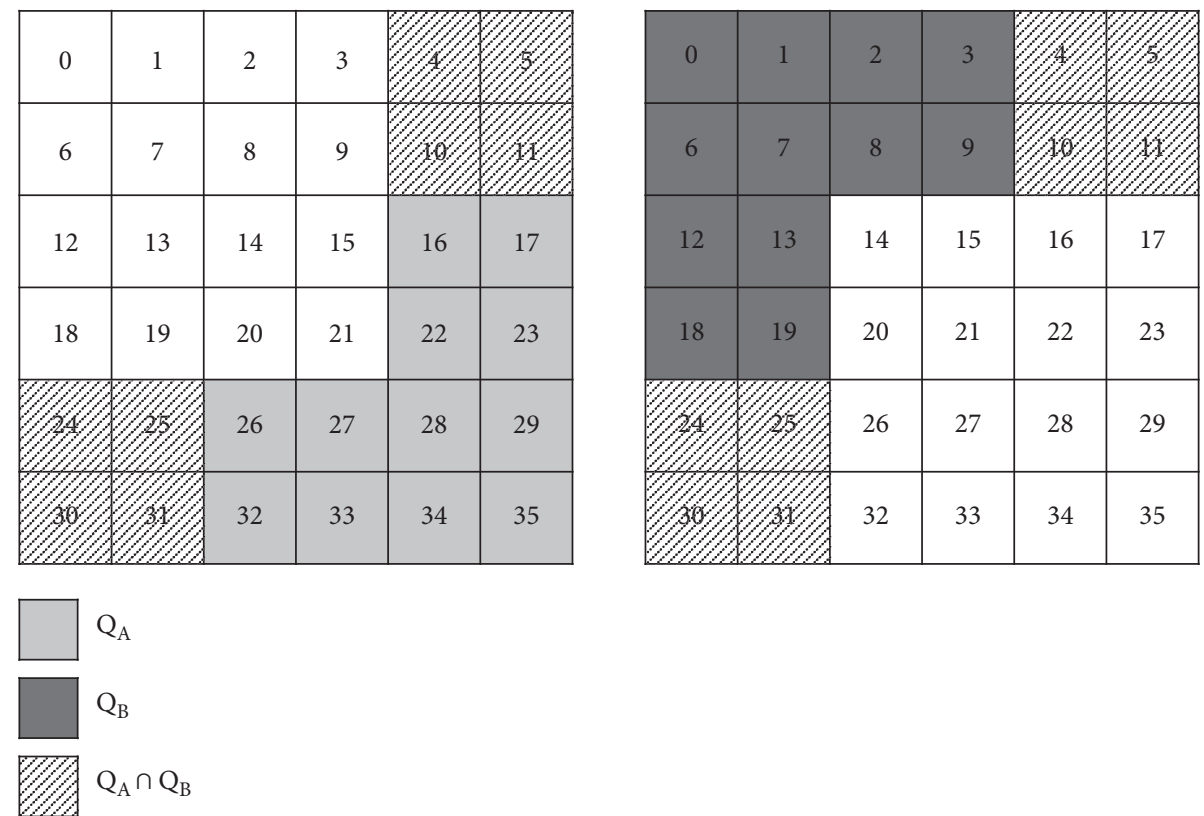

Figure 15: C-Grid quorum system.

\begin{tabular}{|c|c|c|c|c|c|c|c|}
\hline 0 & 1 & & & 4 & 5 & 6 & 7 \\
\hline 8 & 9 & 10 & 11 & 12 & 13 & 14 & 15 \\
\hline 16 & 17 & 18 & 19 & 20 & 21 & 22 & 23 \\
\hline
\end{tabular}

\begin{tabular}{|c|c|c|c|c|c|c|c|}
\hline 0 & 1 & & & 4 & 5 & 6 & 7 \\
\hline 8 & 9 & 10 & 11 & 12 & 13 & 14 & 15 \\
\hline 16 & 17 & 18 & 19 & 20 & 21 & 22 & 23 \\
\hline
\end{tabular}

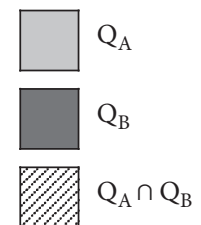

Figure 16: C-Torus quorum system.

grid size $=\left\{\begin{array}{cc}1 \times 1, & \text { if } \mathrm{LD}_{i} \geq \text { Thredshold }_{1}, \\ 2 \times 2, & \text { if } \text { Thredshold }_{1}>\mathrm{LD}_{i} \geq \text { Thredshold }_{2}, \\ 3 \times 3, & \text { if } \text { Thredshold }_{2}>\mathrm{LD}_{i} \geq \text { Thredshold }_{3}, \\ 4 \times 4, & \text { if } \text { Thredshold }_{3}>\mathrm{LD}_{i},\end{array}\right\}$

where $\mathrm{LD}_{i}$ is node $i$ 's traffic load.
According to the environment, Threshold ${ }_{1}$ will be set as the traffic load at which the network is considered to be extremely overloaded. Then, when the traffic load is reduced to $2 n-1 / n^{2}$, the grid size increases to $n \times n$. Accordingly, Threshold $_{2}$ is equal to (Threshold $1 \times 2 \times 2-1 / 2^{2}$ ), and Threshold $_{3}$ is equal to (Threshold $1 \times 2 \times 3-1 / 3^{2}$ ). For example, if the network is considered to be overloaded when nodes' traffic load exceeds more than $12 \mathrm{Kbps}$, then 


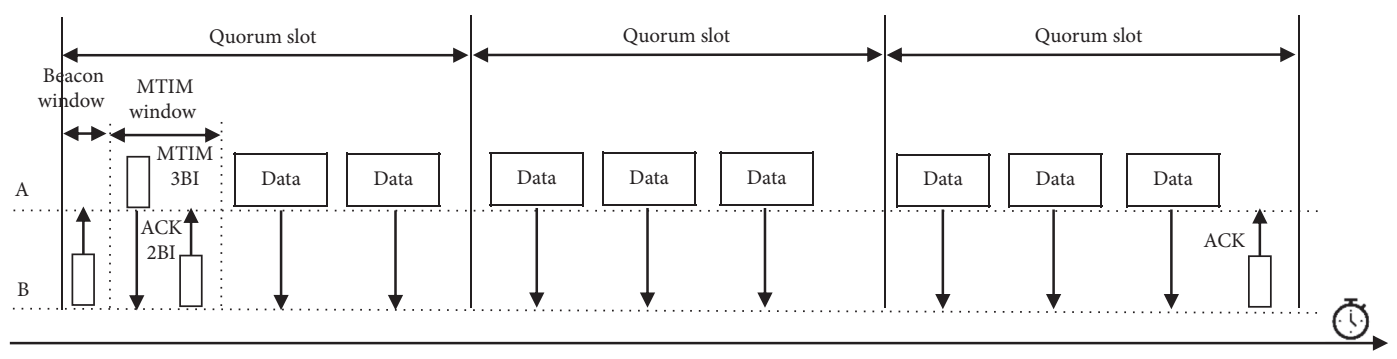

FIGURE 17: Continuous asynchronous power-saving protocol.

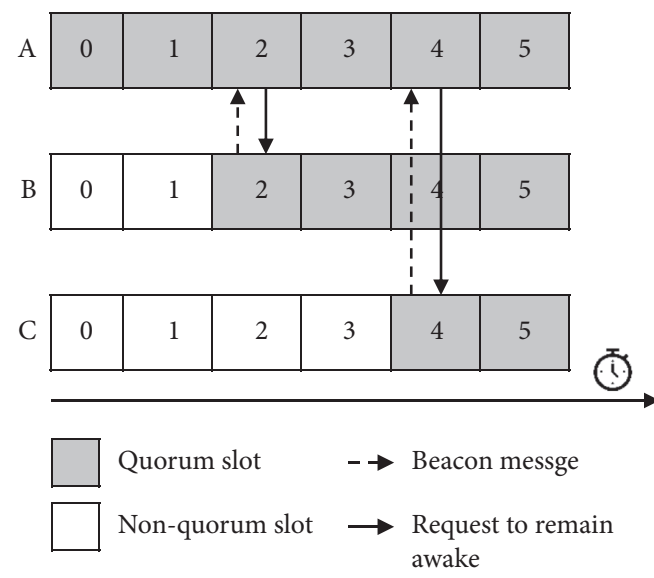

(a)

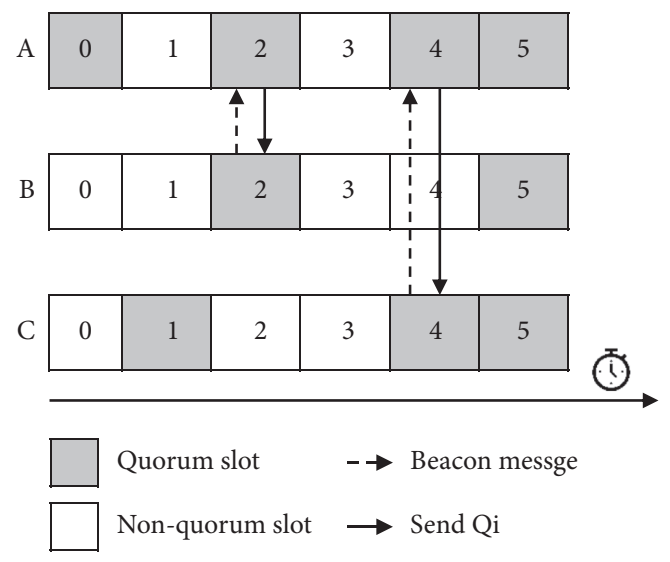

(b)

Figure 18: (a) Busy waiting protocol. (b) Continuous asynchronous multicast power-saving protocol.

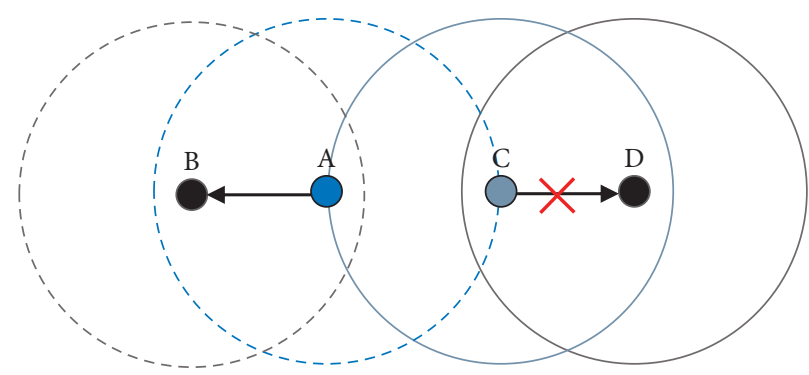

Figure 19: The exposed terminal problem.

Threshold $_{1}=12 \mathrm{Kbps}, \quad$ Threshold $_{2}=12 \times 2 \times 2-1 / 2^{2}$ $=9 \mathrm{Kbps}$, and Threshold $3=12 \times 2 \times 3-1 / 3^{2}=6.67 \mathrm{Kbps}$.

Using the grid quorum system, the network lifetime is improved compared to IEEE 802.11 PSM. This protocol guarantees that any two nodes meet each other two times at least in $n$ slots. However, waiting for the intersecting wakeup slots in order to send a buffered packet will result in increasing the end-to-end delay. To address this problem, the authors propose $\mathrm{AQEC}+$. In AQEC+, if a node has a packet to send, it will stay awake until the packet is sent. As depicted in Figure 20, if A has a packet for B at slot 3, A will remain awake trying to reach its receiver until the packet is sent in slot 5 instead of normally sending it in slot 6 . After sending the packet, node A returns to its quorum-based schedule.
This way, energy consumption may increase, but the protocol will achieve a lower delay. In conclusion, this protocol shows higher delay compared to IEEE 802.11 PSM while achieving higher energy conservation.

3.3.2. Hyper-Quorum System-Based Power-Saving (HQPS) Protocol [17, 18]. HQPS is an IEEE 802.11PSM-based protocol that allows nodes with different requirements to have different cycle lengths while guaranteeing the intersection between any two nodes. By using the hyper-quorum system (HQS), nodes can pick any cycle length that suits their delay and energy constraints. Then, when nodes want to communicate, HQS makes projections of two quorums 

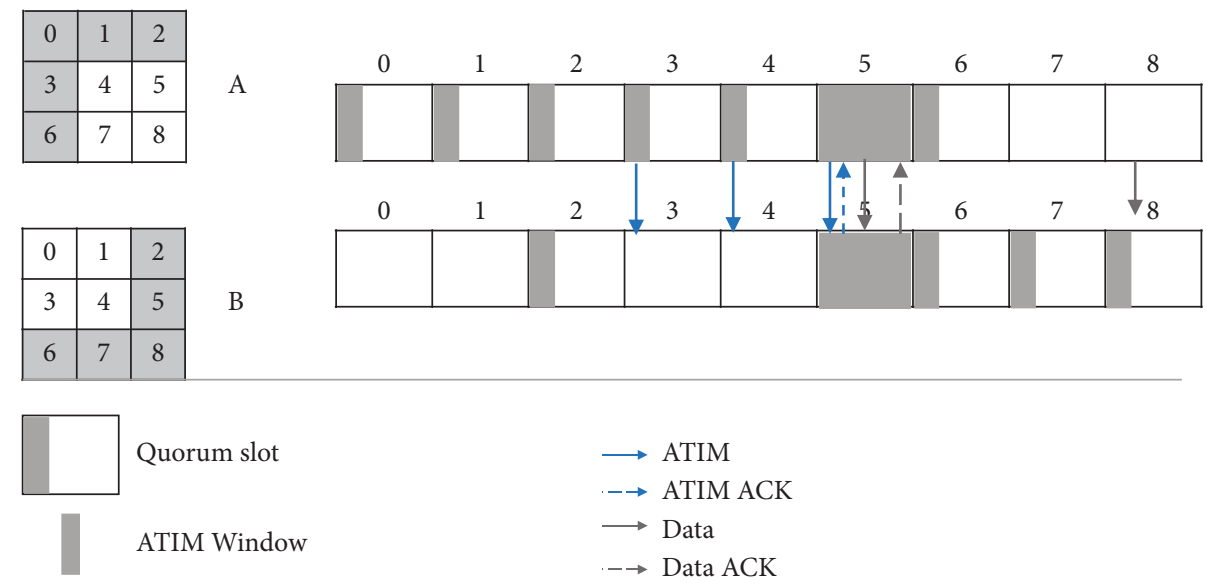

FIGURE 20: AQEC+ protocol.

with different sizes over a module-m plane. HQS guarantees that the projections of quorums intersect at least one $\mathrm{BI}$ in $m$ BIs. Each BI can be a quorum slot or a non-quorum slot. As shown in Figure 21, node A is with initial cycle length $n_{A}=4$ and a quorum set $Q_{A}=\{1,2,3\}$ and node $B$ is with initial cycle length $n_{B}=9$ and a quorum set $Q_{B}=0,3,6,7,8$. A and $B$ quorum slots are guaranteed to intersect at least once within $10 \mathrm{BIs}$, where $m=10$ and the intersecting quorum slots are 5,7,8. This protocol proposes two construction schemes for HQS: difference set (DS) and extended grid (EG). DS scheme produces small-size quorums; accordingly, it is more suitable when the battery power is critical for besteffort traffic, whereas EG scheme suits the delay-sensitive traffic due to its low buffering delay.

Work in [18] is an enhancement of HQPS [17] that proposes two cycle patterns: synchronous cycle pattern and asynchronous cycle pattern. According to the synchronous cycle pattern, a node wakes up in quorum slots where it needs to be awake at the ATIM window only, as illustrated in Figure 22(a). Differently, according to the asynchronous cycle pattern, a node must wake up in the entire quorum slot and in the ATIM window of the first BI after quorum slot, as shown in Figure 22(b). Moreover, an analytic model was proposed to allow nodes to choose a suitable scheme and its cycle length based on delay threshold, drop threshold, and energy incentive. However, these two protocols are vulnerable to ATIM frame collision. To conclude, this protocol increases energy conservation by $31 \%$ compared to AQEC [16].

3.3.3. Adaptive S-Grid (AS-Grid) Quorum System [19]. AS-grid is proposed to allow nodes to adjust its quorum according to its residual energy and node requirements like traffic load. This quorum system is a S-grid system where nodes have the same $t$ value and different $w$ values. According to this quorum system, all nodes must have the same $t$ value in order to guarantee at least one intersection between any two nodes. For example, if A chooses a quorum set from AS-grid $\left(t \times w_{1}\right)$ and B chooses quorum set from AS-grid $\left(t \times w_{2}\right)$, then AS-grid guarantees that $\mathrm{A}$ and $\mathrm{B}$ quorum slots will intersect at least once in $t \times w_{2}$ where $w_{2} \geq w_{1}$. In this adaptive protocol, the node can change its quorum size according to [19] as follows:

$$
\text { grid size }=\left\{\begin{array}{l}
t \times w_{1} \text { if } f-k<\mathrm{RE}<f, \\
t \times\left(w_{1}+1\right) \text { if } f-2 k<\mathrm{RE}<f-k, \\
\cdot \\
\cdot \\
t \times\left(w_{1}+\mathrm{nk}\right) \text { iff }-(n+1) k<\mathrm{RE}<f-\mathrm{nk},
\end{array}\right.
$$

where RE is the remaining energy, $f$ is the full energy, and $k$ is an integer that is derived from network conditions like traffic load.

This protocol is suitable for varied traffic loads. Moreover, it shows better neighbor sensibility compared to the grid and S-grid quorum systems [14]. However, it is assumed that nodes are not synchronized. Thus, nodes have to remain awake during the quorum slot to ensure slot intersection. Even worst, it is vulnerable to control packet collisions when neighbor nodes start communication simultaneously since they follow the IEEE 802.11PSM BI structure. Moreover, like most of the quorum-based power-saving protocols, this protocol increases the end-to-end delay as a result of buffering a data packet and waiting for the intersecting wakeup slots to forward it.

3.3.4. Traffic Load-Based Scheduling (TLS) Protocol [20, 21]. TLS is an energy-efficient quorum-based MAC protocol that schedules node wakeup times using the grid quorum system. Based on the node's traffic load, each node can select its grid size. TLS assumes that (1) nodes are uniformly distributed in an irregular region, are static, have the same transmission distance, and are time-synchronized, (2) the sink is placed at the center of the area, and (3) area is partitioned into equalwidth coronas (zones) based on the hop counts from the sink. TLS network topology is shown in Figure 23. 


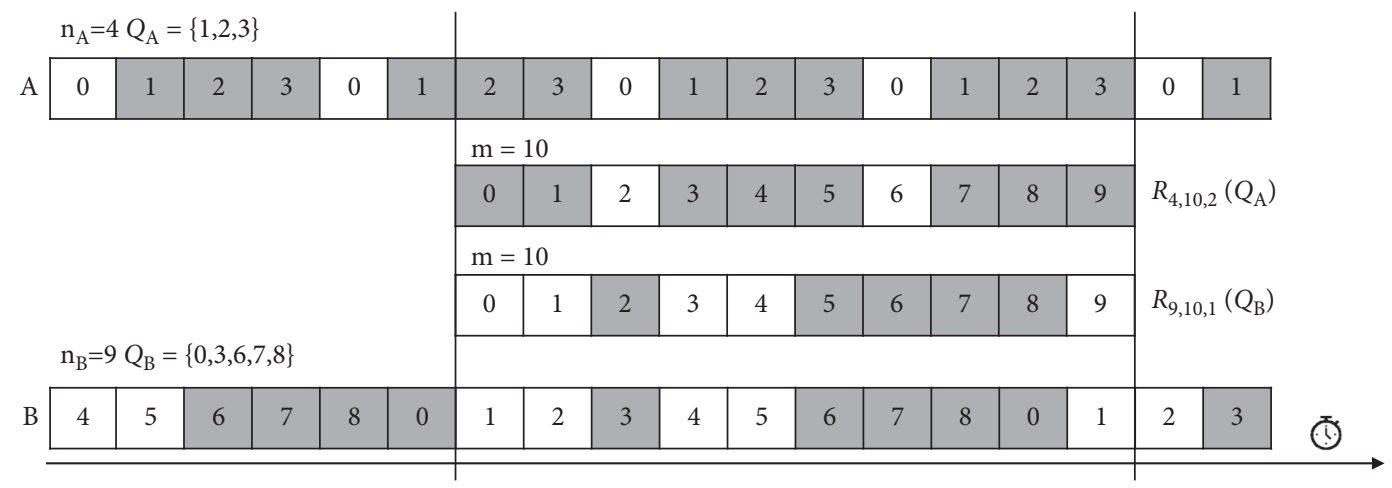

Figure 21: The projections of quorums over modulo-10 plan.

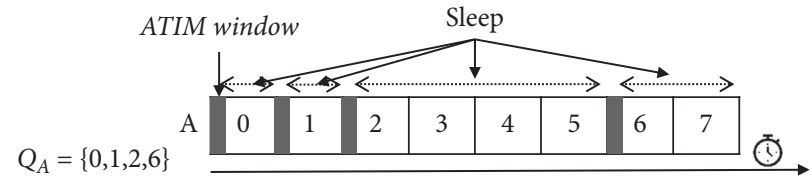

(a)

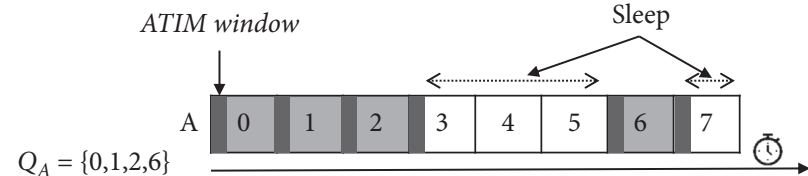

(b)

Figure 22: (a) Synchronous cycle pattern. (b) Asynchronous cycle pattern.

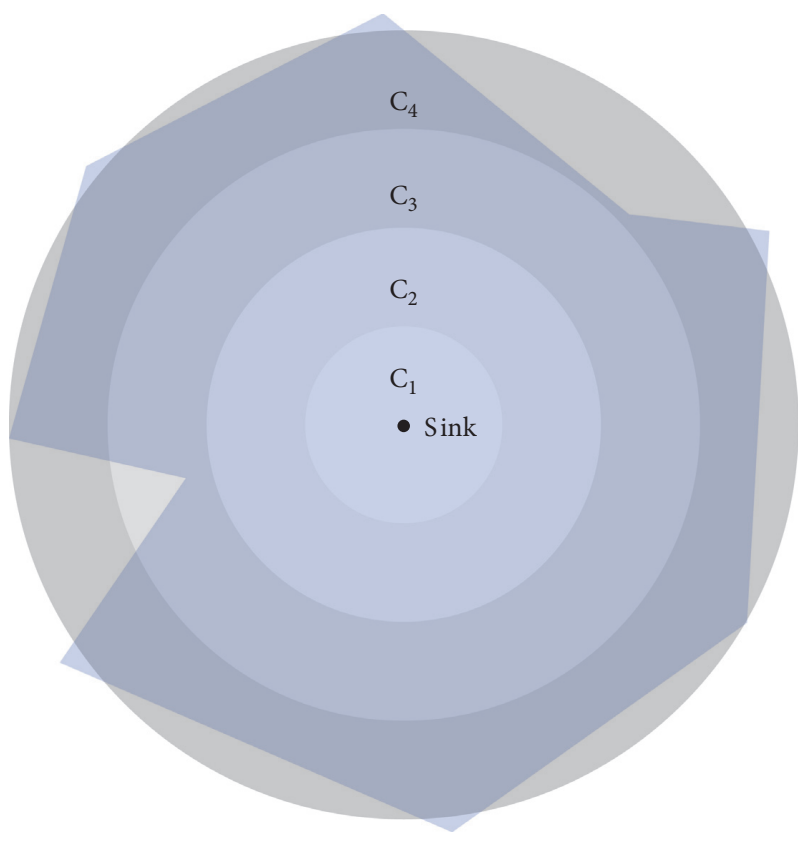

FIGURE 23: TLS network topology.

Initially, every node will determine the size of its grid according to the maximum traffic load it reaches. First, depending on its hop count and the density of its transmission range, every node can determine its traffic load. Node $i$ 's traffic load $\beta_{i}$ [20] is calculated as follows:

$$
\beta_{i}=\frac{\lambda}{D\left(\chi_{i}\right)}\left(1+\frac{1}{1+2\left(\chi_{i}\right) r(i)}\left(1-\chi_{i}^{2}\right)\right),
$$

where $\chi_{i}$ is the distance from the sink to the node $i, r$ is width of the zone, $D\left(\chi_{i}\right)$ is the density at $\chi_{i}$, and $\lambda / D\left(\chi_{i}\right)$ is the data rate transmitted by each node. Then, the node will calculate its active ratio $\gamma_{i}[20]$ as follows:

$$
\gamma_{i}=\frac{\beta_{i}}{\alpha T}
$$

where $\alpha$ is the maximum data rate and T is the cycle length. After that, the node will find the grid size that suits its calculated active ratio, as explained in [20], as follows:

$$
\frac{2 N-1}{N^{2}}=\gamma_{i}
$$

Whenever the node's traffic load changes, it can recalculate its traffic load. Accordingly, it will recalculate its active ratio and find the proper grid size. In this protocol, a path selection algorithm is proposed to determine the nodes' forwarders. In this algorithm, the node collects its next-hop neighbors' residual energy information. When the next-hop neighbors reply, it chooses the next-hop neighbor with the largest amount of residual energy as a forwarder.

In TLS, between any node and its forwarders, at least two intersections are guaranteed. By adjusting nodes' grid size based on their load, this protocol saves more energy and improves network lifetime compared to AQEC [16] and QMAC [30], where the grid size is fixed for nodes in the same corona. However, as in QMAC [30], a collision may occur if two neighbors belonging to the same corona choose the same forwarder and begin sending packet at the same time in the intersecting slot.

3.3.5. Homogeneous MAC (HMAC) Protocol [22]. HMAC, an energy-efficient MAC protocol that minimizes nodes' idle listening time, was proposed. In this protocol, each node selects one or more columns from an $\sqrt{n} \times \sqrt{n}$ 
grid. Then, it can adjust its grid size based on the number of pending packets on its buffer. According to this protocol, the node chooses a number of columns according to four traffic load levels: low, medium, high, or very high traffic load.

This protocol work as follows. First, the queue threshold is assumed to be 12 , and the grid size is assumed to be 16 $(4 \times 4$ grid). If the node's pending packets are 1,2 , or 3 , then the node will choose the first column as its quorum slots, and its active ratio is equal to $\sqrt{n}$. If the node's pending packets are 4,5 , or 6 , then the node will choose the first and the second columns, and its active ratio is equal to $\sqrt{2 n}$. If the node's pending packets are 7,8 , or 9 , then the node will choose the first, the second, and the third columns and its active ratio is equal to $\sqrt{3 n}$. If the node's pending packets are 10,11 , or 12 , then the node will choose all four columns, and its active ratio is equal to $\sqrt{4 n}$.

This protocol allows nodes to adapt their wakeup time based on their pending packet. It guarantees that two nodes will intersect at least 4 times (when nodes choose column one). Moreover, it has better energy efficiency compared to QMAC [30] in one-hop network. However, it imposes a constraint on the cycle length and the chosen columns. Furthermore, it is not suitable for multihop communication.

3.3.6. Adaptive Quorum-Based Power-Saving (AQPS) Protocol [23]. Another adaptive quorum-based power-saving protocol was proposed in [23]. It utilizes the grid quorum system to minimize nodes' idle listening time based on their traffic load. In this protocol, a fixed-column quorum was proposed. According to this protocol, each node can adjust its grid size at the end of each cycle. Each node chooses a random row and a fixed column as its quorum set.

The fixed-column selection is proposed to overcome two non-intersection problems that may appear when choosing different quorum sets on different cycles. The first problem occurs when two neighbor nodes do not have any intersecting slot since they select different rows and columns on each cycle. For example, as shown in Figure 24, A chooses a different row and column from $5 \times 5$ grid on different cycles. Indeed, $A$ chooses $Q_{A 1}$ and then $Q_{A 2}, Q_{A 3}$, and $Q_{A 4}$. B chooses $Q_{B 1}$ from $8 \times 8$ grid. If the first slot in $B$ cycle is the 16th slot in A cycle, then no intersection exists between $A$ and $B$. The second problem happens when two nodes select a different grid size on each cycle, and this may end up with no intersecting slot. As shown in Figure 25, A chooses a different grid size on different cycles. If the first slot in B cycle is the 17th slot in A cycle, then no intersection exists between A and $\mathrm{B}$. The fixed-column selection guarantees that two nodes have at least one intersection. For example, as shown in Figure 26, if A chooses different grid sizes while selecting a fixed column, the second column, then A and B will have intersections. To overcome these two problems, this protocol states that each node may choose an arbitrary row with a mandatory fixed column as its quorum set in each cycle.

This protocol prolongs the network lifetime by utilizing the quorum concept. Moreover, it guarantees the intersection between any two nodes when they are dynamically adjusting different quorum sets. However, since this protocol is based on IEEE 802.11PSM, it suffers from control packet collision.

\subsubsection{Optimal Fully Adaptive and Asynchronous (OFAA)} Protocol [24]. OFAA is designed for multihop MANET to allow any two asynchronous nodes with different cycle lengths to have at least one intersection. This protocol proposed a half-awake beacon interval (HBI) to provide more energy saving and shorter neighbor discovery time. Each HBI is divided into a beacon window (BW), a data window (DW), and another beacon window. At the end of the second BW, a node can enter the sleep state if no communication is needed. In OFAA, the quorum slots will follow the HBI structure and the non-quorum slots will follow the sleep BI (SBI) where the node will be sleeping during the slot time.

This paper proposed a factor-hereditary quorum space. Accordingly, the protocol will generate an HBI/SBI schedule table using this quorum space. The table contains the position of HBIs for the cycle length $S_{i}$ where $1 \leq S_{i} \leq S_{\max }$ and $S_{\text {max }}$ is the maximum cycle length nodes can adjust. Figure 27 shows an example of HBI/SBI schedule table where $S_{\max }=25$. Hence, all nodes store the same HBI/SBI schedule table. According to OFAA, a node can adjust its cycle length at the end of each cycle. Once the node obtains its suitable cycle length, it must look up to the table to get its quorum set. As shown in Figure 28, for example, node A chooses $S_{A}=8$, and node B chooses $S_{b}=6$ and $S_{\max }=25$. According to $\mathrm{HBI} / \mathrm{SBI}$ schedule table shown in Figure 27, $Q_{A}=\{0,1,3,7\}$ and $Q_{B}=\{0,1,3\}$.

OFAA has also proposed an awake/sleep prediction method where the sender can predict when its receiver will be awake. In this prediction method, each beacon message must contain the cycle length and the current BI position in addition to the 802.11 parameters. Each node has to record this information about all its neighbors. Accordingly, when communication is needed, the node looks up at the HBI/SBI schedule table to get receiver schedule. If the receiver is in $\mathrm{SBI}$, the node waits for the receiver coming HBI. If the receiver is in $\mathrm{HBI}$, the node directly sends the data. If the node needs more than a data window, it will set more data bit to 1 , and they will remain awake until their communication is finished. For instance, as shown in Figure 28, A and B discover each other in slot 3 in A's cycle and slot 1 in B's cycle. At slot 7 in A's cycle, if A wants to send a packet to B, it will predict and wait till B's upcoming DW, which is slot 0 in A's cycle, and then send the data.

OFAA saves more energy by utilizing the quorum concept and the HBI structure. Thus, network lifetime is improved compared to AQEC [16] and HQS [17]. Furthermore, to reduce the delay, this protocol utilizes more bits. However, if two nodes have data for the same receiver and start sending simultaneously, their frames will collide.

\subsection{Asymmetric Protocols with Limited Intersection Size}

3.4.1. Asymmetric Cyclic Quorum (ACQ) Protocol [25]. ACQ is a wakeup scheduling protocol designed for clustered ad hoc networks. In the clustered network, a group of nodes 

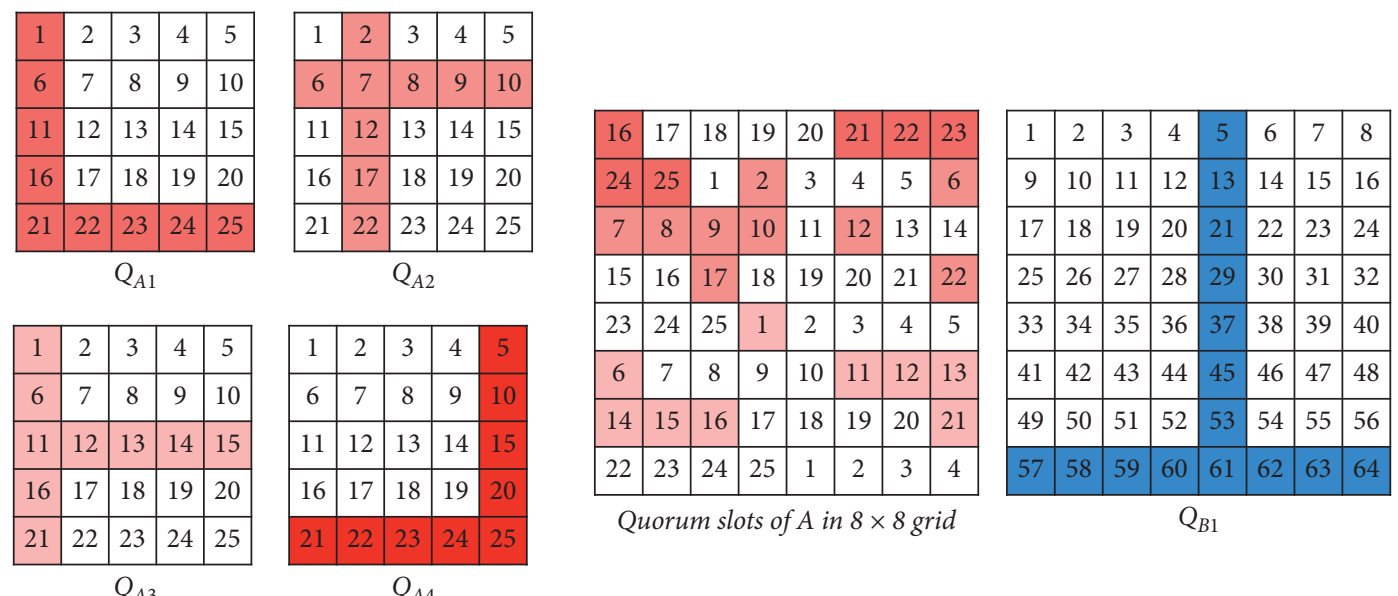

Figure 24: An example of the first problem.
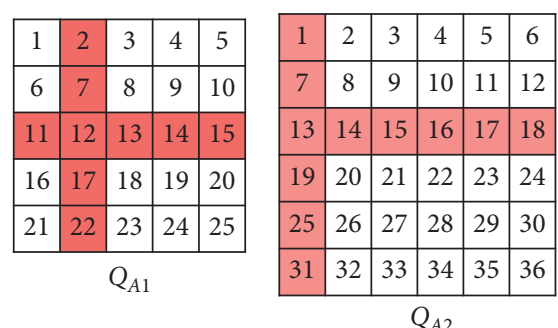

\begin{tabular}{|c|c|c|c|c|c|c|}
\hline 1 & 2 & 3 & 4 & 5 & 6 & 7 \\
\hline 8 & 9 & 10 & 11 & 12 & 13 & 14 \\
\hline 15 & 16 & 17 & 18 & 19 & 20 & 21 \\
\hline 22 & 23 & 24 & 25 & 26 & 27 & 28 \\
\hline 29 & 30 & 31 & 32 & 33 & 34 & 35 \\
\hline 36 & 37 & 38 & 39 & 40 & 41 & 41 \\
\hline 43 & 44 & 45 & 46 & 47 & 48 & 49 \\
\hline \multicolumn{5}{|c|}{$Q_{A 3}$} \\
\end{tabular}

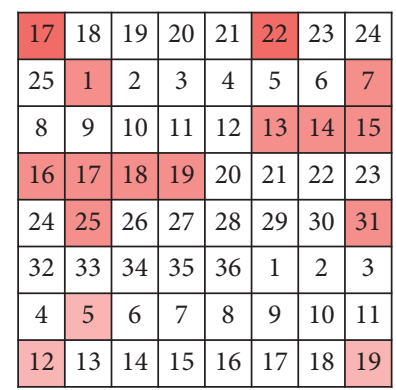

Quorum slots of $A$ in $8 \times 8$ grid

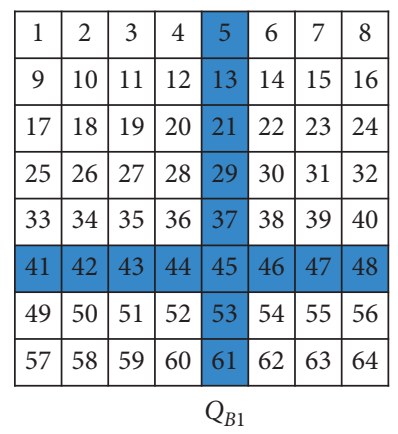

Figure 25: An example of the second problem.
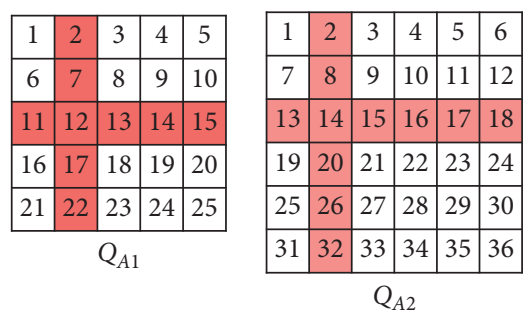

\begin{tabular}{|c|c|c|c|c|c|c|}
\hline 1 & 2 & 3 & 4 & 5 & 6 & 7 \\
\hline 8 & 9 & 10 & 11 & 12 & 13 & 14 \\
\hline 15 & 16 & 17 & 18 & 19 & 20 & 21 \\
\hline 22 & 23 & 24 & 25 & 26 & 27 & 28 \\
\hline 29 & 30 & 31 & 32 & 33 & 34 & 35 \\
\hline 36 & 37 & 38 & 39 & 40 & 41 & 41 \\
\hline 43 & 44 & 45 & 46 & 47 & 48 & 49 \\
\hline \multicolumn{7}{|c|}{$Q_{A 3}$}
\end{tabular}

\begin{tabular}{|c|c|c|c|c|c|c|c|}
\hline 17 & 18 & 19 & 20 & 21 & 22 & 23 & 24 \\
\hline 25 & 1 & 2 & 3 & 4 & 5 & 6 & 7 \\
\hline 8 & 9 & 10 & 11 & 12 & 13 & 14 & 15 \\
\hline 16 & 17 & 18 & 19 & 20 & 21 & 22 & 23 \\
\hline 24 & 25 & 26 & 27 & 28 & 29 & 30 & 31 \\
\hline 32 & 33 & 34 & 35 & 36 & 1 & 2 & 3 \\
\hline 4 & 5 & 6 & 7 & 8 & 9 & 10 & 11 \\
\hline 12 & 13 & 14 & 15 & 16 & 17 & 18 & 19 \\
\hline
\end{tabular}

Quorum slots of $A$ in $8 \times 8$ grid

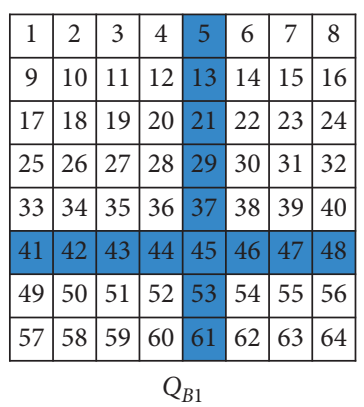

FIGURE 26: An example of the fixed-column selection. 


\begin{tabular}{|c|c|c|c|c|c|c|c|c|c|c|c|}
\hline $\begin{array}{c}\text { Cycle } \\
\text { length }\end{array}$ & \multicolumn{4}{|c|}{ Position of HBIs } & $\begin{array}{c}\text { Cycle } \\
\text { length }\end{array}$ & \multicolumn{7}{c|}{ Position of HBIs } \\
\hline 2 & 0 & 1 & & & 14 & 0 & 1 & 2 & 3 & 7 & \\
\hline 3 & 0 & 1 & & & 15 & 0 & 1 & 2 & 3 & 7 & \\
\hline 4 & 0 & 1 & 3 & & 16 & 0 & 1 & 3 & 7 & 11 & \\
\hline 5 & 0 & 1 & 3 & & 17 & 0 & 1 & 2 & 4 & 12 & \\
\hline 6 & 0 & 1 & 3 & & 18 & 0 & 1 & 3 & 8 & 12 & \\
\hline 7 & 0 & 1 & 3 & & 19 & 0 & 1 & 2 & 6 & 9 & \\
\hline 8 & 0 & 1 & 3 & 7 & 20 & 0 & 1 & 2 & 3 & 6 & 10 \\
\hline 9 & 0 & 1 & 3 & 8 & 21 & 0 & 1 & 3 & 4 & 14 & 16 \\
\hline 10 & 0 & 1 & 3 & 6 & 22 & 0 & 1 & 2 & 5 & 8 & 13 \\
\hline 11 & 0 & 1 & 3 & 5 & 23 & 0 & 1 & 2 & 3 & 7 & 11 \\
\hline 12 & 0 & 1 & 3 & 7 & 24 & 0 & 1 & 2 & 3 & 7 & 15 \\
\hline 13 & 0 & 1 & 3 & 9 & 25 & 0 & 1 & 2 & 3 & 8 & 12 \\
\hline
\end{tabular}

Figure 27: An example of HBI/SBI schedule table.

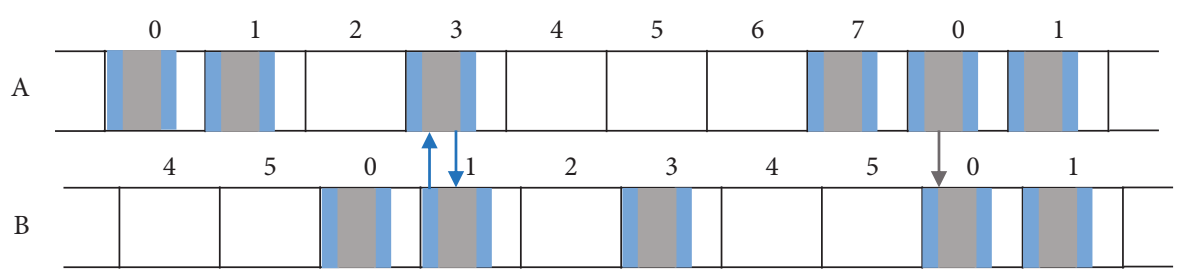

BW

DW

FIgURE 28: An example of OFAA protocol.

forms a cluster. Each cluster has a clusterhead and a gateway. The clusterhead is responsible for forwarding data between members, while the gateway is responsible for forwarding data between clusters. A cluster member only needs to meet clusterhead to forward its data. Accordingly, the clusterhead and the gateway need higher duty cycles than members. For that reason, an asymmetric cyclic quorum system is proposed to allow nodes with different requirements to have different duty cycles (different quorum sizes). This quorum system consists of S-quorum and A-quorum. S-quorum is used by the clusterhead and the gateway and has $k$ as quorum size, while A-quorum is used by cluster members and has $l$ as quorum size, where $l<k$. In quorum-based protocols, a node adjusts its wakeup schedules according to its selected quorum. Here, the quorum slots will follow the IEEE 802.11PSM BI structure. As shown in Figure 29, node A wants to transmit a packet to node $B$. When node $A$ receives node $B$ beacon frame at their overlapping quorum slots, A will send an ATIM frame. Node B sends back an ATIM ACK after it receives the ATIM frame. When A receives ATIM ACK, it starts data transmission. After B received data, it replies with an ACK. On the other hand, if no ATIM ACK is received at the ATIM window, that means a collision has happened, and the node will try to resend the ATIM frame in the next overlapping quorum slot. As in IEEE 802.11PSM, if a node reaches the end of the ATIM window and no ATIM frame is received, the node returns to sleep mode.

The asymmetric cyclic quorum guarantees the intersection among S-quorums and between S-quorum and A-quorum. It guarantees at least one intersection in $n \mathrm{BI}$, even with a BI shift, as shown in Figure 29. Thus, it reduces around $52 \%$ and $36 \%$ of energy consumption compared to AQCE [16] and grid, respectively. However, there is no intersection guaranteed between A-quorums as there is no direct communication among cluster members. This protocol utilizes more bits to reduce the delay by allowing data transmission over multiple consecutive beacon intervals. However, it is vulnerable to collisions when neighbor nodes start communication simultaneously (at the ATIM frames). 


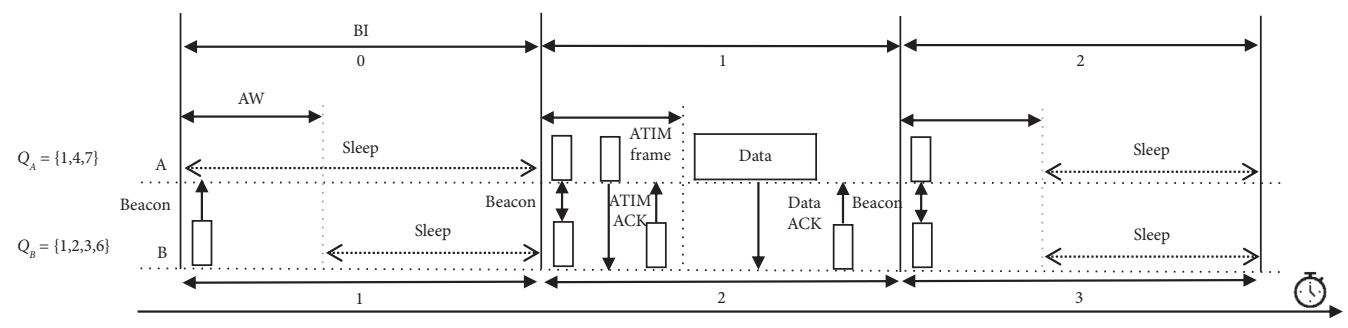

FIgURE 29: An example of ACQ protocol.

3.4.2. Heterogenous Quorum-Based Wakeup Scheduling [26]. In [26], a heterogeneous quorum-based MAC protocol was proposed. In this protocol, two nodes with different cycle lengths ( $n$ and $m$ where $n<m$ ) use two heterogeneous quorums as wakeup schedules. This protocol introduced two quorum system designs: grid quorum system pair (GQSpair) and cyclic quorum system pair (CQS-pair). These quorum systems ensure that two nodes overlap at least once in $m$ slots. Moreover, a construction algorithm was proposed for the fast construction of CQS-pair. The multiplier theorem is used to generate difference sets, and a verification matrix is used to verify that the intersection is not empty. For GQS-pair construction, according to this paper, any two arbitrary grid quorum systems are a GQS-pair. The quorum slot is divided into a beacon window and a data window. This protocol assumed that nodes are not synchronized. Thus, nodes have to remain awake during the quorum slot to ensure slot intersection. A node sends a beacon message to its neighbors to inform them that it is awake. Each node can be either in idle mode or active mode. If there are no data to send or to receive, a node enters the idle mode. At the quorum slot, if the node has data to send or receive, it goes to the active mode. In the active mode, the sender sends the data to its receiver. If the sender has more packets to transmit, it will send, at the end of the data window, a keepawake packet to the receiver. Then, the receiver sends back an acknowledgment to indicate that it will remain awake in the next slot.

This protocol shows better performance in terms of discovery delay compared to the grid quorum system. However, it is limited to two different schedules only. It suffers from beacon packet collisions. Moreover, the node must remain awake throughout its entire wakeup slot which may increase the energy consumption.

3.5. Adaptive Asymmetric Protocols with Fixed Intersection Size. QueenMAC [27] is a quorum-based MAC protocol that aims at minimizing wakeup time of the nodes to save their energy. To enable simultaneous data transmissions, this protocol operates on multiple channels. It utilizes a channel assignment method to reduce collisions. According to QueenMAC, the sink is placed at the corner, and nodes are assumed to be uniformly distributed. Moreover, nodes are time-synchronized and grouped based on their hop counts between them and the sink. For instance, nodes in group $G_{i}$ are $(i+1)$ hops away from the sink. QueenMAC network topology is shown in Figure 30.
The dygrid quorum system is used by QueenMAC to schedule nodes' wakeup time. There are two quorums in the dygrid quorum system: H-quorum and V-quorum. It is defined as dygrid $\left(r_{1}, r_{2}, k_{1}, k_{2}\right)=H\left(r_{1}, k_{1}\right) \cap V\left(r_{2}, k_{2}\right)$ where $1 \leq k_{1}, k_{2} \leq \sqrt{n}, 1 \leq r_{1}, r_{2} \leq n-1$, and $k_{1}, k_{2}$ are two integers obtained from the traffic load of the nodes. The $k_{1}$ and $k_{2}$ values affect the number of quorum slots. Indeed, larger values of $k_{1}$ and $k_{2}$ lead to more quorum slots. Therefore, nodes near the sink, that have a higher traffic load, can select larger $k_{1}$ and $k_{2}$ which are further away. Each node determines its quorum based on its chosen $k_{1}$ and $k_{2}$ value and arbitrary $r_{1}$ or $r_{2}$ value and determines its quorum type (H-quorum or $\mathrm{V}$-quorum) based on its group number. For example, nodes with even group numbers choose $\mathrm{V}$-quorum, and nodes with odd group numbers choose H-quorum. Accordingly, two neighbor nodes will select a different quorum type as the data forwarding will be limited only to neighbor nodes belonging to consecutive groups. Since nodes in the same group are not supposed to communicate, the dygrid quorum does not guarantee intersections between them. However, it ensures that two neighbors belonging to different groups have $k_{1} \times k_{2}$ intersecting slots in each cycle.

To reduce collisions, each group of nodes selects four different channels: two for broadcast messages and two for unicast messages. One channel is used to receive broadcast packets from the closest group to the sink, and one channel is used to transmit broadcast packets to further group. Furthermore, QueenMAC utilizes two unicast channels: one to receive data packets from the further group and one to forward data packets through the closer upstream group towards the sink. This channel assignment procedure provides two-hop channel separation for every group such that different packet types do not collide. For example, nodes in the farthest group $G_{4}$ will be assigned channels 2 and 4 for receiving and sending broadcast messages, respectively. Similarly, they will be assigned channels 1 and 3 for receiving and sending unicast messages, respectively.

According to QueenMAC, communication is only restricted between nodes in different groups. In other words, nodes in group $G_{i}$ can only receive broadcast and unicast from nodes in group $G_{i-1}$ and $G_{i+1}$, respectively. Moreover, they can only forward broadcast and unicast messages to nodes in group $G_{i+1}$ and $G_{i-1}$, respectively. Each quorum time slot in QueenMAC is divided into mini-control slots and data slot. Each group $G_{i}$ has at most $i+2$ mini-control slots, where $i$ is the group number. Each node chooses three mini-control slots: $i-1, i$, and $i+1$ slots. Figure 31 illustrates 


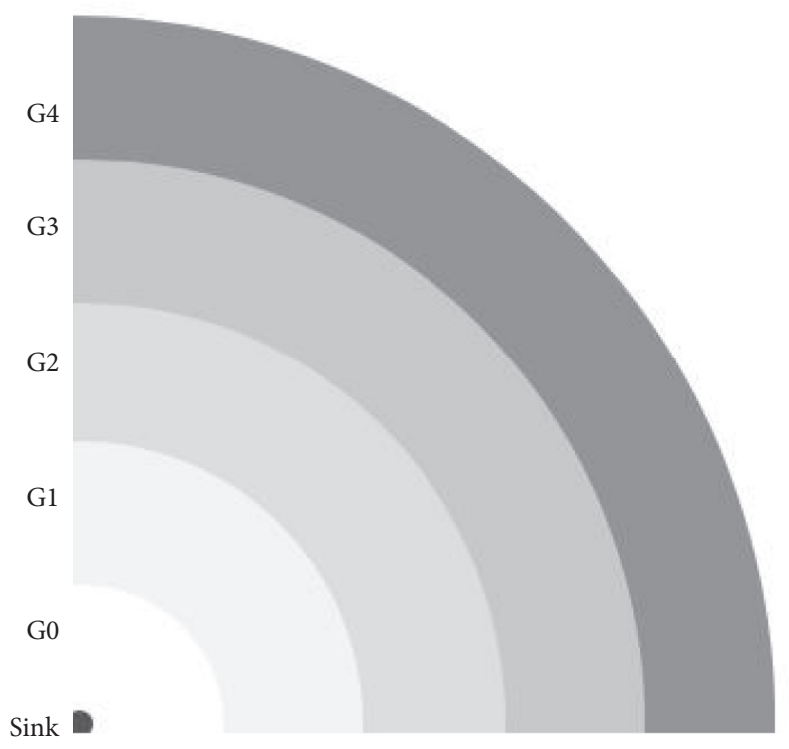

FIGURE 30: QueenMAC network topology.

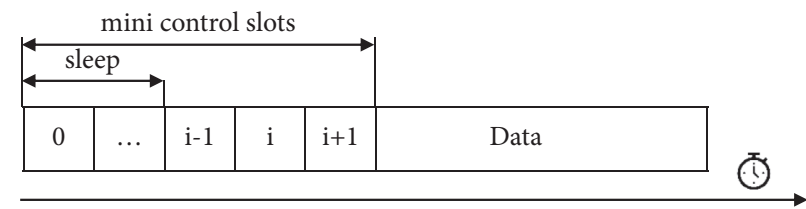

Figure 31: QueenMAC quorum time slot structure.

the structure of the quorum time slot. QueenMAC data communication is explained as follows:

(i) At mini-control slot $(i-1)$, the node wakes up and turns to its receiving broadcast channel. If the channel is busy, the node will receive the broadcast packet until the end of the quorum time slot. Otherwise, the node waits until the start of the next mini-control slot.

(ii) At mini-slot $i$, if the node has a broadcast packet to transmit, it switches to its sending broadcast channel and transmits the broadcast packet until the end of the quorum time slot. If there is no broadcast data to send and the node has a data packet to forward to group $(i-1)$, it switches to the sending unicast channel. The node sends RTS to group $(i-1)$. Nodes that have received the RTS wait for a random backoff time before sending CTS. This backoff time is chosen such that the node with high remaining energy will reply first. If the CTS is received, the node sends a data packet during the quorum time slot.

(iii) If there is no data packet to send or no received CTS, at mini-control slot $(i+1)$, node switches to its receiving unicast channel. The node waits for RTS during this mini-slot period. If RTS is received, the node waits for the backoff time and then replies with a CTS. After that, it waits for the data packet until the end of the quorum time slot.

(iv) Otherwise, after the mini-control slot $(i+1)$, the node goes back to sleep till the next quorum slot.

QueenMAC avoids the possible collision between broadcast and unicast messages using different channels. However, a collision may occur if two nodes belonging to the same group choose the same forwarders from group $G_{i-1}$ and start sending unicast data at the same time in the intersecting quorum slot. As shown in Figure 32, node A and B choose the same forwarders E, F, and G. If they start transmitting RTSs simultaneously, their RTSs will collide at $\mathrm{E}, \mathrm{F}$, and $\mathrm{G}$ and the communication will be impossible. However, if the nodes have just some common forwarders, the non-common ones will receive the request correctly and complete the communication if they are awake (A and $\mathrm{C}$ in Figure 32). Furthermore, a collision may take place when two forwarders have nearly the same remaining energy. Thus, their CTSs will collide. Nevertheless, QueenMAC prolongs the network lifetime and increases the packet delivery rate by using multiple channels. Moreover, the number of intersections between neighbor nodes in the dygrid quorum system increases, which reduces the forwarding delay. However, this protocol suffers from the aforementioned collisions. In addition, QueenMAC nodes can adjust their schedule when their traffic load changes, 


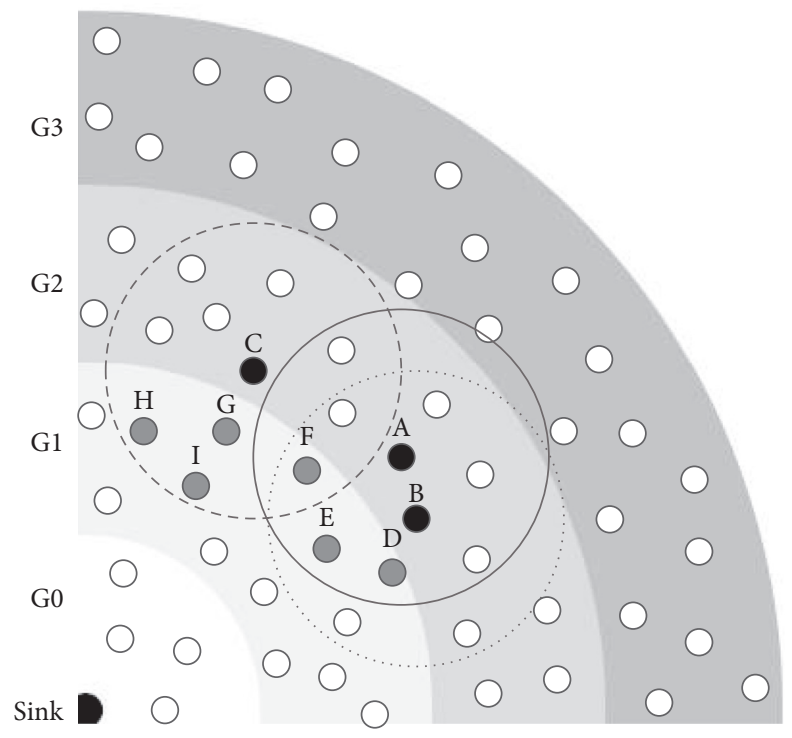

FIGURE 32: Next-hop forwarders.

unlike QMAC [30], where nodes cannot adjust their schedules dynamically.

\subsection{Adaptive Asymmetric Protocols with Limited Intersection Size}

3.6.1. Asynchronous, Adaptive, and Asymmetric (AAA) Protocol [28]. AAA is a power management protocol designed for clustered ad hoc networks. This protocol allows asynchronous nodes to have different cycle lengths with different duty cycles while ensuring the intersection between any two nodes. AAA provides asymmetric quorums by utilizing the asymmetric grid quorum system. The asymmetric grid quorum system is inherited from AQS [25], where a node can select S-quorum or A-quorum depending on its requirements. A node with S-quorum will randomly pick a row and a column from $\sqrt{n} \times \sqrt{n}$ grid. A node with A-quorum will randomly select a column from $\sqrt{n} \times \sqrt{n}$ grid. Moreover, AAA provides adaptive cycle length by guaranteeing the intersection between nodes with different grid sizes. To strike a balance between energy and delay constraints, AAA suggests for a given cluster's nodes to adjust their cycle lengths together. Accordingly, when cluster load exceeds a predetermined threshold, the clusterhead requests cluster members to shrink their grid size to reduce the buffering delay. In addition to the quorum slot and non-quorum slot, AAA proposed a monitor beacon interval (MBI) where a node needs to be awake during the ATIM window. AAA proposed two cycle patterns types: delay-sensitive cycle pattern and best-effort cycle pattern. In the delay-sensitive cycle pattern, quorum elements represent the quorum slots and other BIs will be MBIs, as shown in Figure 33(a). At ATIM window of MBI or quorum slot, if an ATIM frame is received, the node remains awake the entire BI for data transmission. As a result, the buffering delay will decrease. However, the energy consumption will increase. In the best-effort cycle pattern, BI can be a quorum slot, a non- quorum slot, or MBI. As shown in Figure 33(b), after quorum slot, there is a MBI. As in ACQ [25], AAA uses ATIM frame, ATIM ACK, data, and data ACK for communications where ATIM frames may collide and nodes are required to retransmit the ATIM frame in the next quorum slot. Moreover, unlike ACQ and HQPS, a node must be awake during the entire quorum slot to ensure the intersection between asynchronous nodes.

3.6.2. Asymmetric and Asynchronous Energy Conservation Protocol [29]. In [29], an improvement of ACQ [25] was proposed with the objective of minimizing the duty cycles for clustered vehicular networks. An asymmetric majority quorum (AMQ) was proposed to allow nodes to adapt their wakeup schedules according to their delay requirements. In this clustered network, a group of vehicles moving in the same direction will form a cluster. AMQ consists of two quorums: A-quorum and S-quorum. The construction of AMQ is based on $\beta$, which denotes the maximum delay that is allowable in asymmetric links (vehicle-to-vehicle (V2V) links), and $\alpha$, which denotes the maximum delay that is allowable in symmetric links (vehicle-to-roadside (V2R) or V2V links). A-quorum is used by cluster members with $A$ $(\alpha)=\{0\}$, while $\mathrm{S}$-quorum is used by clusterheads, gateways, and road side units (RSUs) with card $(S(\alpha, \beta))=\alpha-1 / 2$. As in HQPS [18], to allow node communications, a projection of two quorums is made over a module-m plane, where $m=$ $\alpha-1$ when the communication is between S-quorums and $m=\beta-1$ when the communication is between an A-quorum and a $\mathrm{S}$-quorum. An example where $\alpha=12$ and $\beta=9$ is shown in Figure 34.

This protocol guarantees at least one intersection within $\beta$ BIs between S-quorums and at least one intersection within $\beta \mathrm{BIs}$ between $\mathrm{S}$-quorum and A-quorum. It achieves higher energy efficiency compared to the grid quorum system. However, it does not guarantee the intersections between A-quorums. Moreover, it suffers from ATIM frame collisions. 


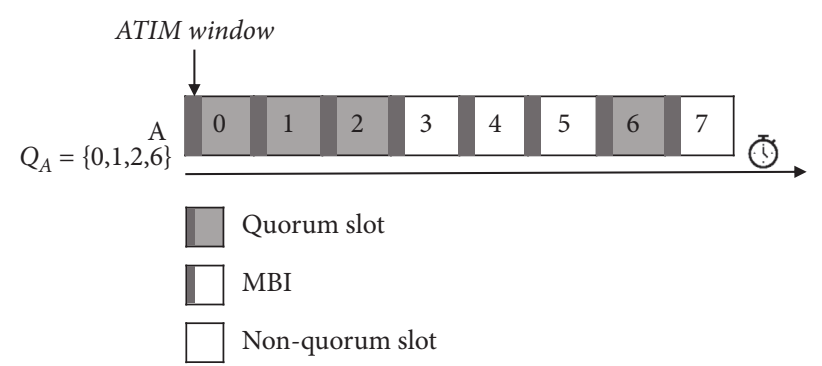

(a)

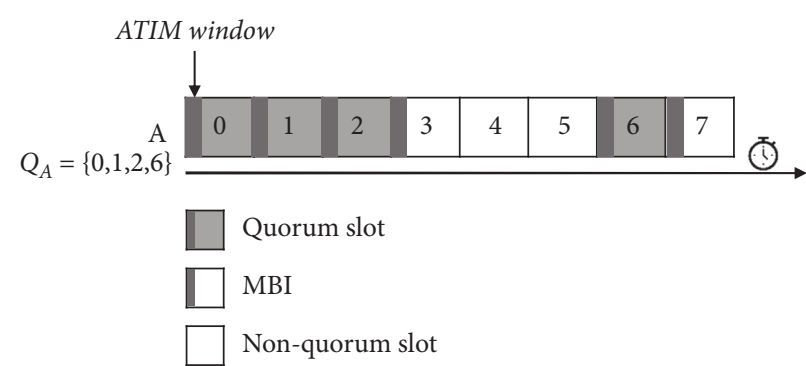

(b)

Figure 33: (a) Delay-sensitive cycle pattern. (b) Best-effort cycle pattern.

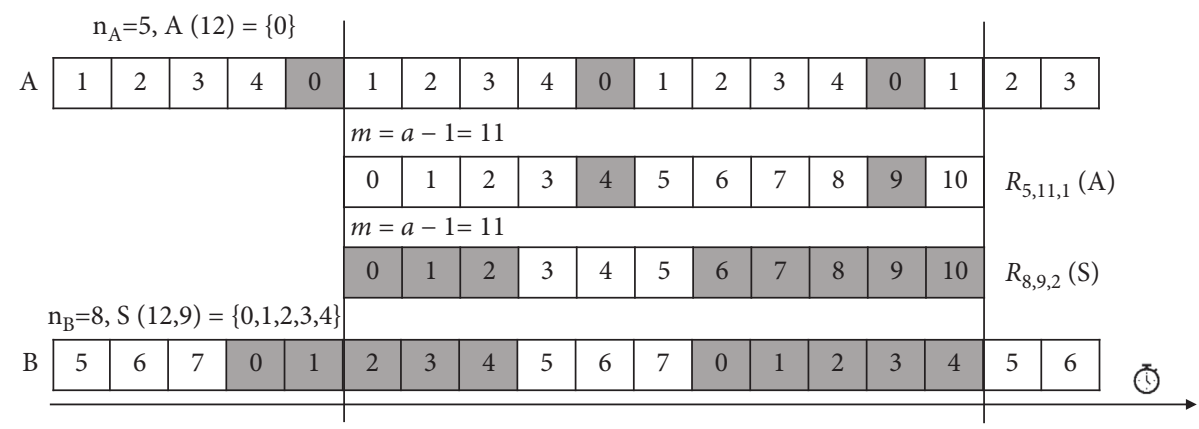

(a)

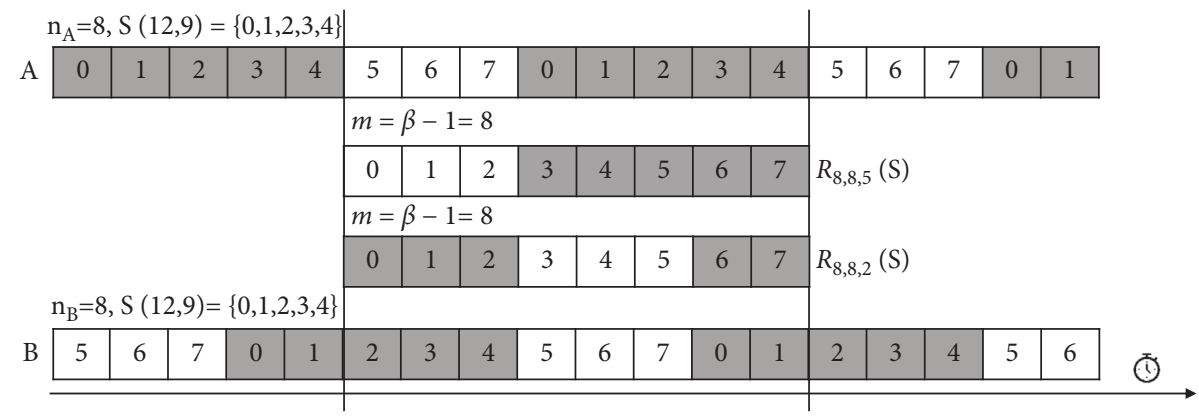

(b)

Figure 34: (a) The projection of $A(12)$ and $S(12,9)$ on module-11 plane. (b) The projection of $S(12,9)$ and $S(12,9)$ on module-8 plane.

3.6.3. Quorum-Based MAC (QMAC) Protocol [30]. QMAC is an energy-efficient MAC protocol that was designed for minimizing nodes' idle listening time based on their traffic load. Moreover, it proposed the next-hop group selection process to reduce network latency that is resulting from nodes' long sleep time. QMAC assumes that (1) nodes are uniformly distributed, are static, have the same transmission distance, and are time-synchronized, (2) area is partitioned into equal-width coronas, (3) the sink is located at the center, and finally (4) each node has a unique ID. QMAC network topology is shown in Figure 35. In order to create the coronas, at the initialization phase, the sink sends a broadcast message containing a hop count field. Each node increments this field by one and the message is rebroadcast. According to QMAC, the message delivery to the sink has to cross upstream corona (corona with a smaller ID) till reaching the sink.
The grid quorum system is used by QMAC to schedule nodes' wakeup times. Each node has a grid size that is different from its neighbors in the upstream corona. Each node randomly chooses one row and one column from its grid as its quorum times. During the non-quorum time, the node goes to sleep state to save energy. At the quorum time, a node has to remain awake for one-fifth of the length of the quorum time. If the channel is busy, the node remains awake to receive data. Otherwise, the node enters the sleep mode. QMAC frame structure is depicted in Figure 36. The grid quorum system guarantees that two nodes meet each other at least two times.

According to QMAC, the farther the corona from the sink, the larger its grid size as such nodes tend to have smaller activity ratio (duty cycle) than the closer ones. Each node determines its grid sizes based on its traffic load. Nodes in the inner corona, near to the sink, are heavily loaded. 


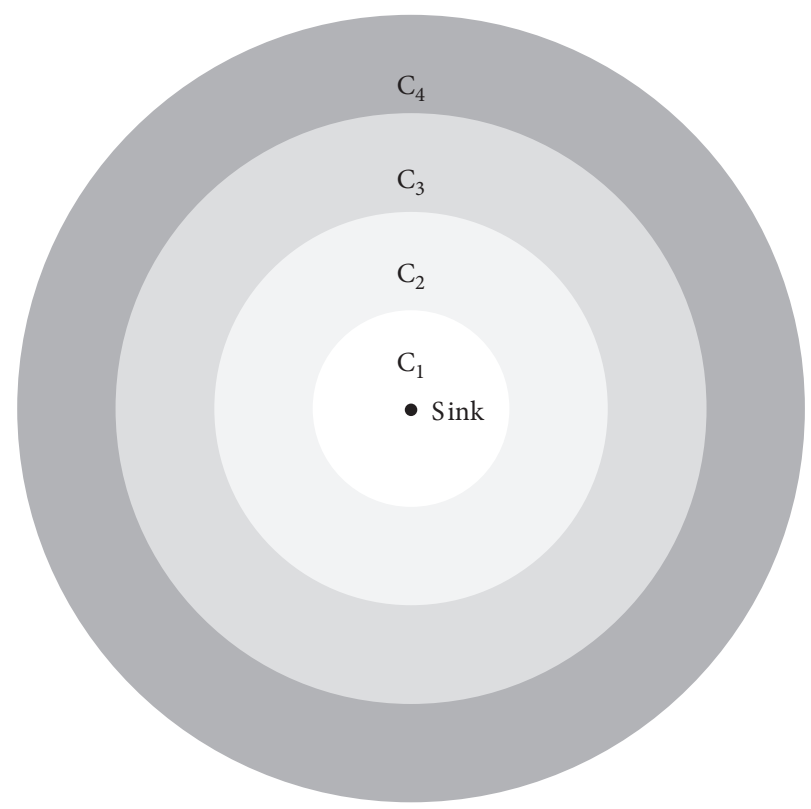

FIGURE 35: QMAC network topology.

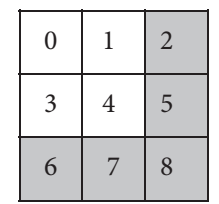

A

\begin{tabular}{|l|l|l|l|l|l|l|l|l|}
\hline 0 & 1 & 2 & 3 & 4 & 5 & 6 & 7 & 8 \\
\hline
\end{tabular}

\begin{tabular}{|l|l|l|}
\hline 0 & 1 & 2 \\
\hline 3 & 4 & 5 \\
\hline 6 & 7 & 8 \\
\hline
\end{tabular}

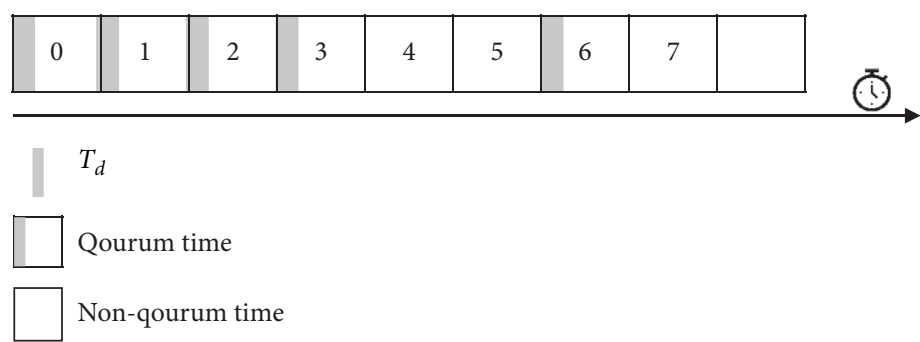

FIGURE 36: QMAC frame structure.

These nodes choose a small grid size to obtain a high active ratio. On the other hand, nodes in the outer corona choose a larger grid size. The first corona grid size will be chosen based on the reporting rate. Then, the other coronas will choose their grid size based on the traffic load ratio between coronas. The node traffic load [30] is calculated as follows:

$$
T_{C_{i}}=1+\frac{\left|C_{i+1}\right|}{\left|C_{i}\right|} \times T_{C_{i+1}} .
$$

For example, in a four-corona network $\left(C_{1}, C_{2}, C_{3}\right.$, and $C_{4}$ ), the traffic load will be $16,5,2.4$, and 1 , respectively. If the first corona $C_{1}$ uses a $2 \times 2$ grid, its active ratio will be 0.75 . Accordingly, the active ratio for $C_{2}, C_{3}$, and $C_{4}$ will be $0.234(0.75 \times 5 / 16), 0.112$, and 0.047, respectively. Each corona will select the grid size that satisfies its active ratio. In this case, the grid size for $C_{2}, C_{3}$, and $C_{4}$ must be $8 \times 8$, $17 \times 17$, and $42 \times 42$, respectively.

QMAC reduces the end-to-end latency by not only using a different grid size (activity ratio) but also by allowing the node to rely on more than one node to forward its traffic to the sink. This group of nodes is called the next-hop forwarder group. The group member must be in the node's coverage and in the upstream corona $C_{i-1}$. In order to create the next-hop group, at the initialization phase, each node selects a number of nodes randomly as its forwarders. The next-hop group size [30] can be determined using the following:

$$
\text { pi }=1-\left(\frac{n_{g}-1}{n_{g}}\right)^{2 \times n_{h}} \text {, }
$$


where pi is the probability for a node in grid $C_{i}$ to find at least one of its next-hop forwarders awake, assuming that nodes in $C_{i}$ have $n_{g} \times n_{g}$ grid, and $n_{h}$ is the size of the next-hop group. The next-hop group size depends on $p i$ and activity ratio (grid size) of nodes in the upstream corona $C_{i-1}$. For instance, the next-hop group size for a node in $C_{2}$ is equal to 2 if the desired awake probability is 0.9 and the grid size of node in $C_{1}$ is $2 \times 2$.

In order to enable communications between sensor nodes, QMAC follows the four-way handshaking. As shown in Figure 37, assume node A wants to forward its packet to the sink. A has 3 members in its next-hop group B, C, and D. First, it sends a multicast RTS packet to its next-hop group members. The group members wait for a random backoff time before sending CTS. This backoff time is chosen such that the node with high remaining energy will reply first. C replies first with the CTS, as shown in Figure 37. Then, when A receives CTS, it waits for Short Interframe Space (SIFS) before transmitting its data packet to C. Finally, when $C$ receives the data correctly, it waits for SIFS before replying with an ACK. In this scenario, node $\mathrm{E}$ is not a member of A's next-hop group. Thus, it ignores the RTS packet and goes back to sleep even if it receives the RTS.

In QMAC, two possible collision scenarios may happen. The first scenario happens when two neighbors from the same corona select the exact same forwarder group and start sending data at the same time in the intersecting quorum slot. As shown in Figure 38, node A and B choose the same next-hop group members E, F, and G. If they start transmitting RTSs simultaneously, their RTSs will collide at E, F, and $\mathrm{G}$ and the communication will be impossible. However, if the nodes have just some common group members, the non-common ones will receive the request correctly and complete the communication if they are awake ( $\mathrm{A}$ and $\mathrm{C}$ in Figure 38). The second scenario takes place when two nodes from the same next-hop group set their backoff time on the same contention period because they have nearly the same remaining energy. Thus, their CTSs will collide. The probability of this scenario depends on the size of the next-hop forwarder group and the size of the contention window.

QMAC prolongs the network lifetime while decreasing network latency by utilizing the concept of the next-hop forwarder group along with different grid size. It increases energy conservation and successful delivery ratio compared to DMAC [3] and PMAC [8] (non-quorum-based protocols). However, this protocol may suffer from collisions in the aforementioned situations. Moreover, when a node selects its next-hop group, it may choose less energy-efficient nodes as its group members.

3.6.4. Quorum Time Slot Adaptive Condensing (QTSAC) Protocol [31]. QTSAC is a quorum-based MAC protocol that was designed with the objective of minimizing the delay and maximizing the energy efficiency. In this protocol, quorum slots are allocated according to nodes' data transmission periods. In addition, QTSAC attempts to reduce the network latency by adding more quorum slots depending on the residual energy of nodes. QTSAC assumes that (1) nodes are uniformly distributed, time-synchronized, and grouped into rings based on their distance from the sink, (2) the sink is in the center of the area, (3) the width of the ring is less than the transmission distance of the nodes, and (4) nodes transmit data in multihop using the aggregation model. According to this protocol, the quorum slot is divided into a beacon window and data window, as shown in Figure 39. If a node has a message to send, it will send a beacon message to its intended receiver. Receiving a beacon message means that a data packet will be received during the data window. Thus, if a node receives a beacon message, that means a data packet will be received during the data window and it remains awake in the entire quorum slot. Otherwise, the node enters the sleep mode.

QTSAC MAC protocol works as follows. First, the quorum time slots are condensed during the nodes' data transmission periods. To do so, this protocol uses the SOgrid quorum system to generate a $(\sqrt{n}-x) \times(\sqrt{n}-y)$ condensed grid from a $\sqrt{n} \times \sqrt{n}$ grid, where $n$ is cycle length and $(x, y)$ pair represents the number of excluded rows and columns from the original grid, as shown in Figure 40. SOgrid quorum system consists of two quorums: S-quorum and $\mathrm{O}$-quorum. As in QueenMAC, each node selects S-quorum or $\mathrm{O}$-quorum based on its ring number. If the ring number is even, a node can choose S-quorum, and if it is odd, a node can choose $\mathrm{O}$-quorum. For instance, node $\mathrm{A}$ selects random value $m_{1}$ and its neighbor node B selects random value $m_{2}$, where $1 \leq m_{1}, m_{2} \leq(\sqrt{n}-x) 2 / \sqrt{n}$. According to SO-grid quorum construction, node $\mathrm{A}$ has $m_{1}$ slots in each column on its condensed grid and node $\mathrm{B}$ has $m_{2}$ rows containing all its quorum slots. Thus, node $\mathrm{A}$ and node $\mathrm{B}$ will have at least $m_{1} \times m_{2}$ intersection slots. Then, a node locates the condensed matrix on the original grid based on its data transmission period. In the data collection network, data are forwarded from far groups to the sink. Accordingly, first, if a node is far from the sink where data transmission takes place on the first half of the cycle $n$, in this case, the condensed grid is placed in the upper-left corner of the original grid, as depicted in Figure 40(b). Second, if a node is near to the sink where data transmission takes place on the second half of the cycle $n$, the condensed grid is in the lower-right corner of the original grid, as depicted in Figure 40(c). Finally, if a node is in the middle of the network, the condensed grid is in the middle of the original grid, as depicted in Figure 40(d). After that, each node adds some quorum slots depending on the remaining energy, which means that nodes far from the sink will be adding more quorum slots than others. Finally, each node starts working according to its quorum wakeup schedule.

QTSAC increases network throughput and minimizes the delay while it prolongs the network lifetime. Moreover, the increase of the quorum slots number in the far area from the sink will further decrease the delay in this area. This protocol performs better in terms of energy efficiency and end-to-end delay compared to the grid quorum system. However, this increase leads to a higher duty cycle in the low traffic load area, which means low energy efficiency. As in QueenMAC, QTSAC did not provide a solution for collisions. In addition, if the node still has more data packets, it 


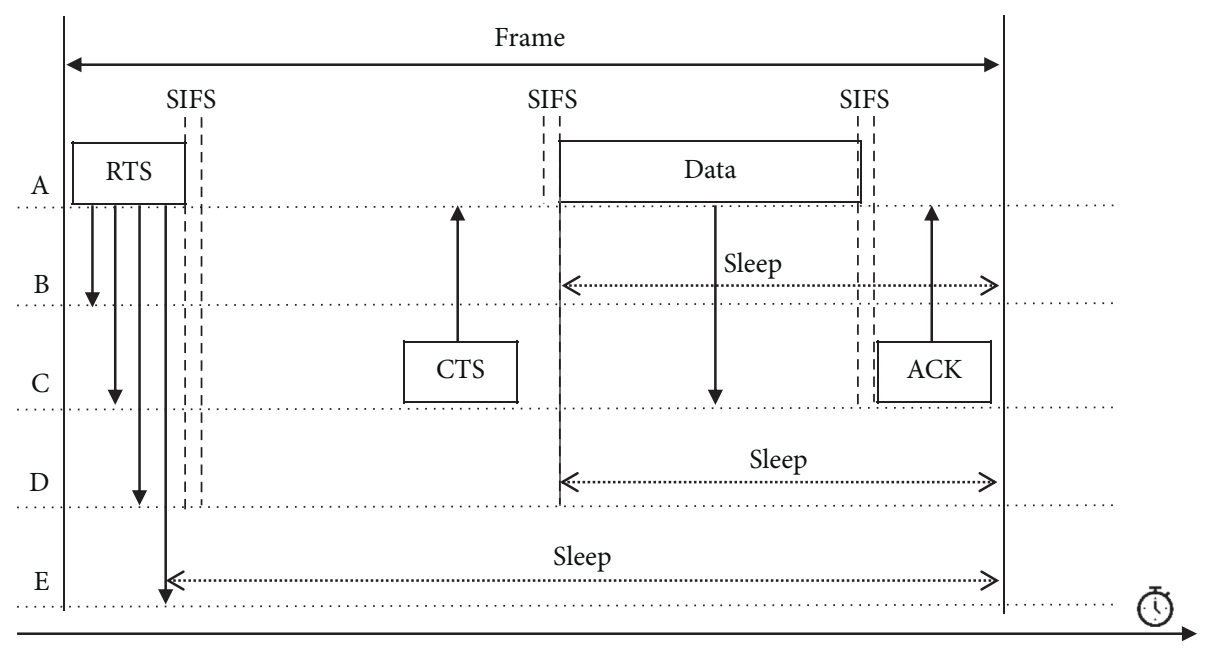

FIgURE 37: QMAC data communication.

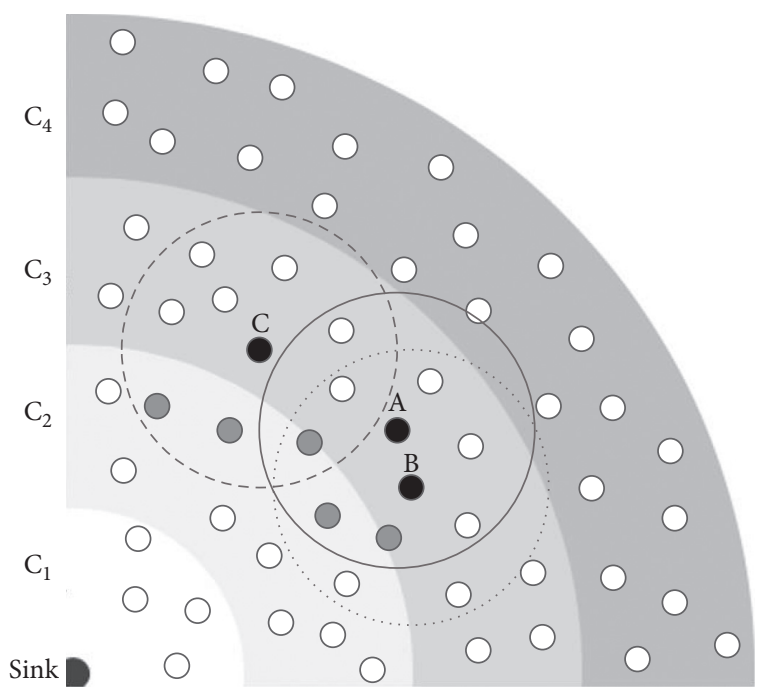

Figure 38: Next-hop group.

A

\begin{tabular}{|c|c|c|}
\hline 0 & 1 & 2 \\
\hline \multicolumn{3}{|c|}{ Beacon window } \\
\hline & \multicolumn{2}{|c|}{ Qourum slot } \\
\hline & Non-qo & $\mathrm{n}$ slot \\
\hline
\end{tabular}

Figure 39: The frame structure of QTSAC.

will wait until the next overlapping slot with its receiver in order to transmit the packet which increases the end-to-end delay, and hence the network throughput will deteriorate.

3.6.5. Element Shift Quorum (ESQ)-Based MAC Protocol [7]. A quorum-based MAC protocol that is designed for Internet of Things (IoT) was proposed in [7]. This protocol assumes that (1) the network is divided into rings, (2) the sink is in the center, and (3) nodes are uniformly distributed, time-synchronized, and grouped into rings based on their distance from the sink. As in QTSAC, in this protocol, quorum slots are allocated according to nodes' data transmission periods. To do so, this protocol uses the ST-grid quorum system to select a number of quorum slots from $w \times \sqrt{n}$ grid that is condensed from a $\sqrt{n} \times \sqrt{n}$ grid, where $n$ is cycle length and $\mathrm{w}$ represents the number of excluded rows from the original grid, as shown in Figure 41. ST-grid quorum system consists of two quorums: S-quorum and T-quorum. Each node selects $\mathrm{S}$-quorum or T-quorum based on its ring number. If the ring number is even, a node can choose S-quorum, and if it is odd, a node can choose T-quorum. For instance, node A selects random value $m_{1}$, where $1 \leq m_{1} \leq w$, and its neighbor node $\mathrm{B}$ selects random value $m_{2}$, where $1 \leq m_{2} \leq \sqrt{n}$. According to ST-grid quorum construction, node $\mathrm{A}$ has $m_{1}$ rows selected from the grid and node B has $m_{2}$ columns selected from the grid. Thus, node $\mathrm{A}$ and node $\mathrm{B}$ will have at least $m_{1} \times m_{2}$ intersecting slots. After each node builds its quorum, a node locates the $w \times \sqrt{n}$ grid on the original grid based on its data transmission period. Accordingly, first, if a node is far from the sink where data transmission takes place on the first half of the cycle $n$, in this case, the node chooses the first $w$ rows from the original grid to place its quorum slots, as depicted in Figure 41(b). Second, if a node is near to the sink where data transmission takes place on the second half of the cycle $n$, the node chooses the last $w$ rows from the original grid to place its quorum slots, as depicted in Figure 41(c). Finally, if a node is in the middle of the network, the node placed its quorum slots in the middle of the original grid, as depicted in Figure 41(d). Once a node places its quorum slots, it starts working according to its quorum wakeup schedule.

This protocol increases network throughput and minimizes the delay while it prolongs the network lifetime. This protocol performs better in terms of end-to-end delay compared to the grid quorum system. As in QueenMAC and QTSAC, it did not provide a solution for collisions.

3.6.6. Multiclass Quorum System (MCQS) [32]. A generalized quorum system named MCQS was proposed for 


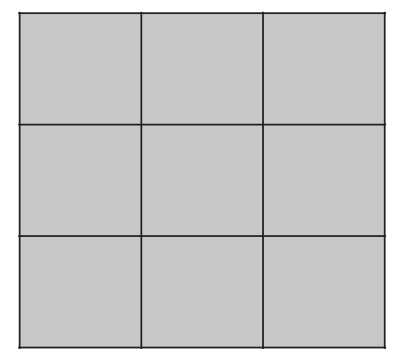

(a)

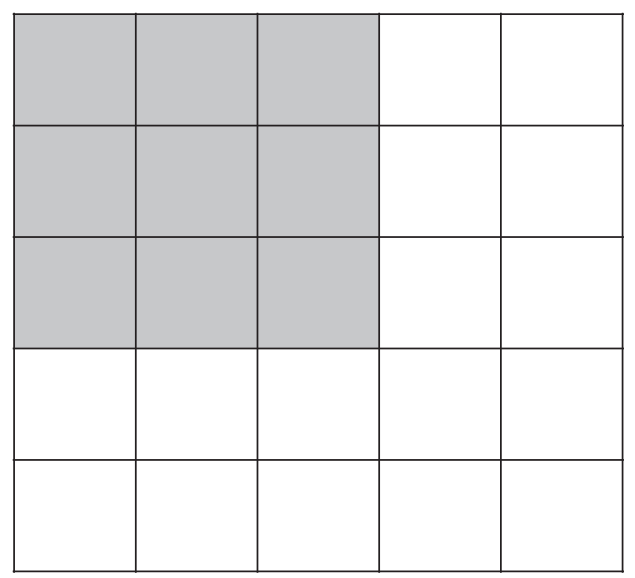

(b)

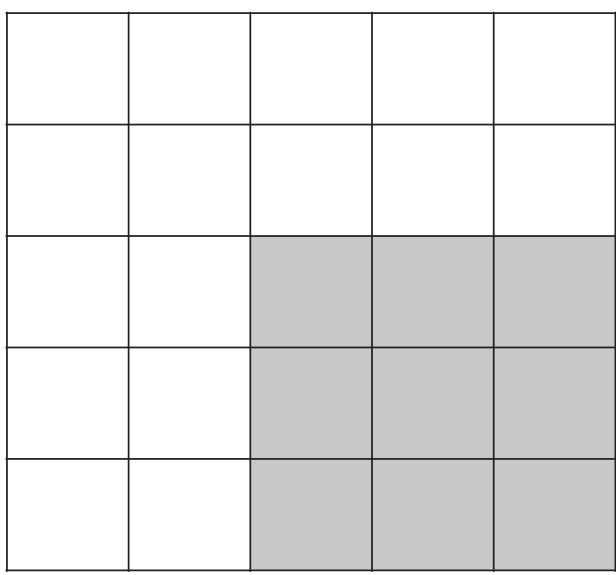

(c)

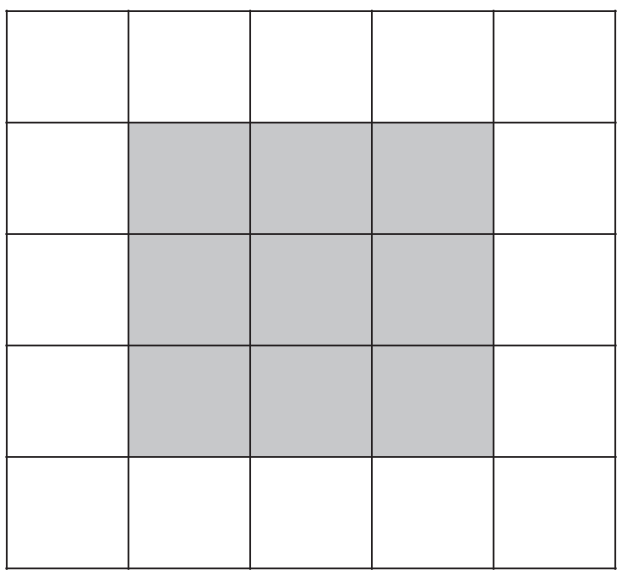

(d)

Figure 40: The condensed grid allocation. (a) Condensed grid. (b) Condensed grid allocation for far node. (c) Condensed grid allocation for near node. (d) Condensed grid allocation for middle node.

asynchronous nodes in multihop WSNs. MCQS is comprised of multiple quorum classes (quorum systems). It ensures a certain number of intersections among quorums in the same class (specific intraclass overlapping size) and a certain number of intersections between quorums in different classes (specific interclass overlapping size). MCQS can be customized to provide any quorum system type. For instance, let $m$ be the number of the classes, $C_{i}$ be the number of intersections specified between quorums in class $i$, and $C_{i, j}$ be the number of intersections specified between class $i$ quorums and class $j$ quorums. If $m=1$ and $C_{1}=2$, this is equivalent to the grid quorum system, where at least two intersections between any two arbitrary quorums are guaranteed. For example, if $m=2, C_{1}=1, C_{2}=0$, and $C_{1,2}=1$, this system will work as the ACQ [25] where class 1 represents the S-quorum and class 2 represents the A-quorum. Furthermore, a binary integer programming model is proposed to define the quorums of each class in MCQS while ensuring the minimum activity ratios for classes.

To allow nodes to choose the best quorum class and the best route to the sink, a quorum-based sleep-scheduling and routing model is proposed. In this model, first, a logical graph is built upon the network topology. Figure 42(a) shows the logical graph of four nodes. Then, the extended graph is constructed on top of the logical graph, where quorums from different classes represent the vertices and each quorum vertex is connected to all its neighbors' vertices. Figure 42(b) shows the extended graph construction, where $v_{i, k}$ is the vertex that represents class $k$ quorum of node $i$. Here, every node will be virtually duplicated into $k$ nodes, where $k$ is the number of possible quorum classes. An edge between $v_{i, k}$ and $v_{j, m}$ represents the possible communication between $v_{i}$ and $v_{j}$, if $v_{i}$ uses class $k$ and $v_{j}$ uses class $m$. Finally, a minimum-weight vertex-disjoint path algorithm is run on the extended graph to obtain the best source to sink routes which will naturally select the best class for each node. By selecting the best quorum class and the best route to the sink, this protocol shows better performance in terms of end-to-end delay and energy efficiency compared to HQPS [17].

\section{Comparison and Research Direction}

In Table 2, we compared the quorum systems used in the reviewed protocols according to the following: 
$\sqrt{n}$

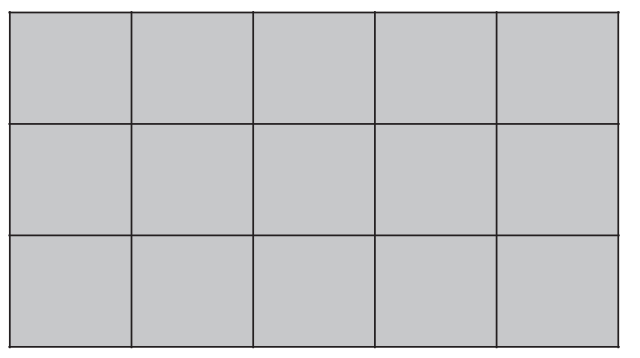

(a)

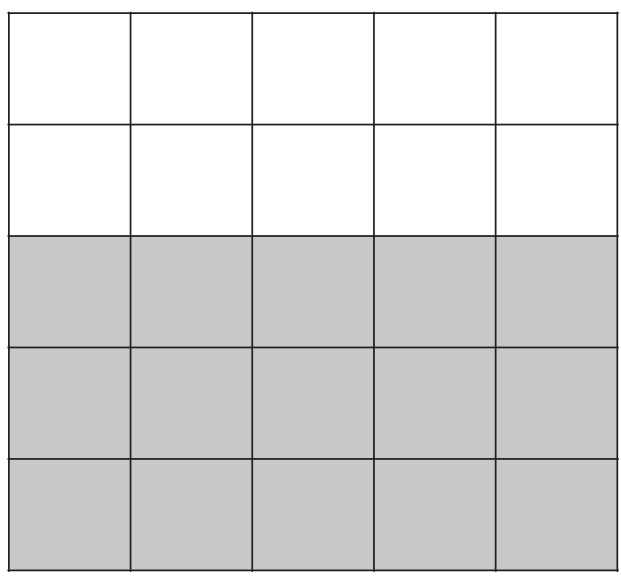

(c)

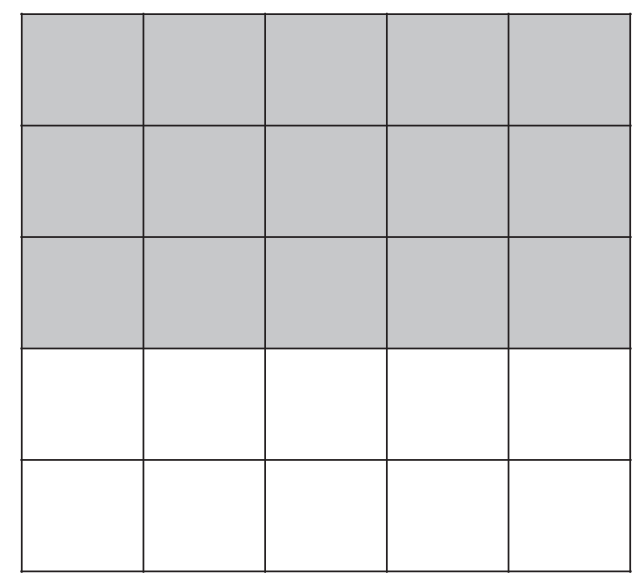

(b)

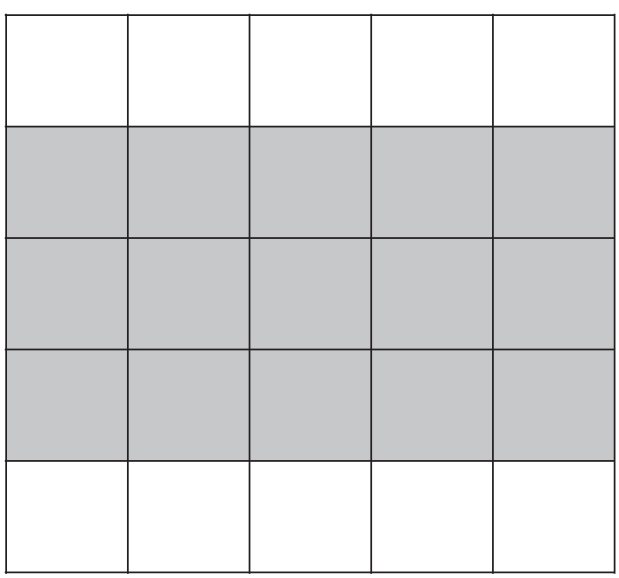

(d)

Figure 41: $w \times \sqrt{n}$ allocation. (a) $w \times \sqrt{n}$ grid. (b) $w \times \sqrt{n}$ grid allocation for far node. (c) $w \times \sqrt{n}$ grid allocation for near node. (d) $w \times \sqrt{n}$ grid allocation for middle node.

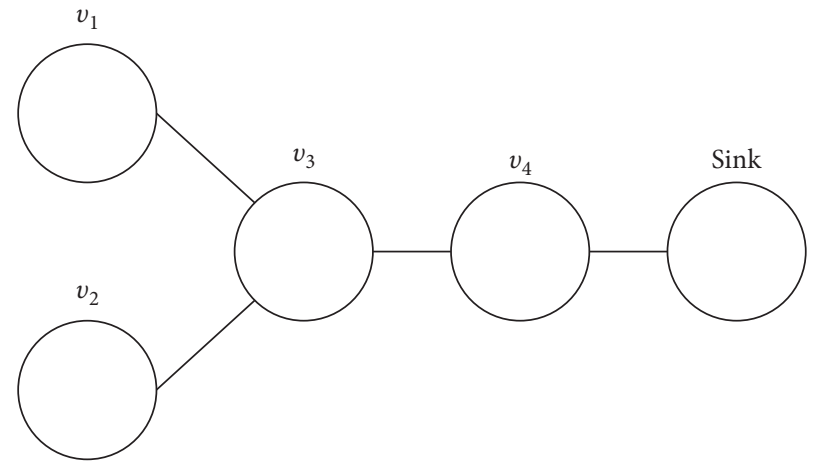

(a)

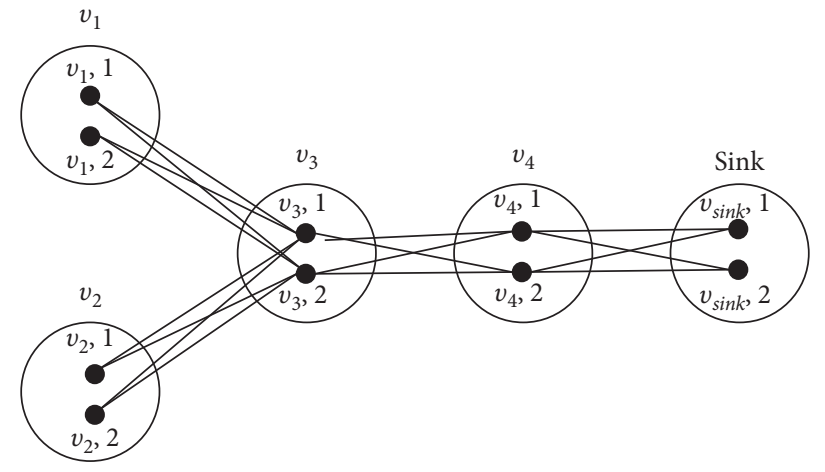

(b)

FIgURE 42: (a) The logical graph. (b) The extended graph.

(i) Active ratio: it is the ratio of the wakeup slots and the cycle length. In fact, the smaller the active ratio, the higher the energy efficiency. In some protocols, nodes have the same activity ratio. Hence, the node may wake up more than its need which reduces the end-to-end delay but decreases the energy efficiency. On the other hand, a node may wake up less than its need and thus increase the delay but improve the energy efficiency. Therefore, several researchers proposed protocols that allow nodes to adapt their active ratio to their conditions like traffic load or delay. 
TABLE 2: Quorum system characteristics of the reviewed protocols.

\begin{tabular}{|c|c|c|c|c|c|}
\hline Protocol & Quorum system & Active ratio & System size (cycle length) & $\begin{array}{c}\text { Guaranteed } \\
\text { intersection size }\end{array}$ & $\begin{array}{l}\text { Quorum slot } \\
\text { assignment }\end{array}$ \\
\hline LAPS [12] & $\begin{array}{l}\text { Location-aware } \\
\text { quorum system }\end{array}$ & Same for all nodes: $6 / 21$ & $\begin{array}{c}\text { Fixed } \\
21\end{array}$ & 1 & Location-based \\
\hline $\begin{array}{l}\text { QMMAC } \\
{[13]}\end{array}$ & $\begin{array}{l}\text { Line quorum } \\
\text { system }\end{array}$ & $\begin{array}{c}s \times l / n \text {, where } s \text { is the number of } \\
\text { slots in each line, } l \text { is number of } \\
\text { wakeup lines, and } n \text { is total } \\
\text { number of lines }\end{array}$ & $\begin{array}{l}\quad n \times s \\
\text { Fixed for all nodes }\end{array}$ & $\begin{array}{l}\text { At least one line slot } \\
\text { and at most } n \text { lines }\end{array}$ & $\begin{array}{c}\text { Location-based } \\
\text { line assignment } \\
\text { for nodes in last } \\
\text { corona }\end{array}$ \\
\hline S-grid [14] & S-grid $(t \times w)$ & $t+w-1 / t \times w$ & $\begin{array}{l}t \times w \\
\text { Fixed for all nodes }\end{array}$ & At least 2 & Randomly \\
\hline \multirow[t]{2}{*}[15]{} & C-grid & $\begin{array}{c}s \sqrt{n}+d \sqrt{n}-\mathrm{sd} / n, \text { where } n \text { is the } \\
\text { system size }\end{array}$ & Same for all nodes & At least $(d \times s) \times 2$ & Randomly \\
\hline & $\begin{array}{l}\text { C-torus } \\
\text { Grid with }\end{array}$ & $s \times t+w / 2-s+2 / t \times w$ & $t \times w$ & At least 2 & Randomly \\
\hline AQEC [16] & $\begin{array}{l}\text { adaptive grid size } \\
\text { based on fixed } \\
\text { traffic load } \\
\text { thresholds }\end{array}$ & $2 \sqrt{n}-1 / n$, where $1 \leq n \leq 4$ & $\begin{array}{l}1,4,9, \text { or } 16 \text { based on } \\
\text { corona traffic load }\end{array}$ & At least 2 & Randomly \\
\hline $\begin{array}{l}\text { HQPS } \\
{[17,18]}\end{array}$ & $\begin{array}{l}\text { HQS (EGHQS } \\
\text { and DSHQS) }\end{array}$ & $\begin{array}{c}\text { Each node has a different activity } \\
\text { ratio }\end{array}$ & $\begin{array}{l}\text { Each node can select its } \\
\text { cycle length }\end{array}$ & At least 1 & Randomly \\
\hline AS-grid [19] & AS-grid $(t \times w)$ & $\begin{array}{c}t+w-1 / t \times w \text {, where } w \text { can be } \\
\text { different for each node }\end{array}$ & $\begin{array}{l}t \times w \text {, where } w \text { can be } \\
\text { different for each node }\end{array}$ & At least 1 & Randomly \\
\hline TLS $[20,21]$ & $\begin{array}{l}\text { Grid with } \\
\text { adaptive grid size } \\
\text { based on node } \\
\text { traffic load }\end{array}$ & $\begin{array}{l}2 \sqrt{n}-1 / n, \text { where } n \text { can be } \\
\text { different for each node }\end{array}$ & Based on node traffic load & At least 2 & Randomly \\
\hline HMAC [22] & $\begin{array}{l}\text { Grid with } \\
\text { adaptive grid size } \\
\text { based on node } \\
\text { pending packet }\end{array}$ & $\begin{array}{c}\sqrt{n}, \text { if } 1 \leq \mathrm{pp} \leq 3 \sqrt{2 n} \text {, if } \\
4 \leq p p \leq 6 \sqrt{3 n} \text {, if } 7 \leq p p \leq 9 \sqrt{4 n} \text {, if } \\
10 \leq \mathrm{pp} \leq 12, \text { where pp is the } \\
\text { number of pending packets }\end{array}$ & $\begin{array}{l}\text { Based on node pending } \\
\text { packet }\end{array}$ & At least 2 & Randomly \\
\hline AQPS [23] & $\begin{array}{l}\text { Adaptive grid } \\
\text { with one } \\
\text { arbitrary row } \\
\text { with one } \\
\text { mandatory fixed } \\
\text { column }\end{array}$ & $\begin{array}{l}2 \sqrt{n}-1 / n, \text { where } n \text { can be } \\
\text { different for each node }\end{array}$ & Based on node traffic load & At least 2 & Randomly \\
\hline OFAA [24] & $\begin{array}{l}\text { Factor-hereditary } \\
\text { quorum space }\end{array}$ & $\sqrt{S_{i}} / S_{i}((1 / 2)+(\mathrm{BW} / \mathrm{BI}))$ & $\begin{array}{c}1 \leq S_{i} \leq S_{\max } \text { and } S_{\max } \text { is } \\
\text { the maximum cycle } \\
\text { length nodes can adjust } \\
\text { and } S_{i} \text { is the chosen cycle } \\
\text { length by node } i\end{array}$ & At least 1 & Randomly \\
\hline ACQ [25] & $\begin{array}{l}\text { AGQ (A-quorum } \\
\text { and S-quorum) }\end{array}$ & $\begin{array}{l}\sqrt{s} / s \text { for cluster member and } \\
2 \sqrt{s}-1 / s \text { for clusterhead, where } s \\
\text { is the system size }\end{array}$ & Same for all nodes & $\begin{array}{l}\text { At least } 1 \text { among } S \text { - } \\
\text { quorum and at least } \\
1 \text { between S-quorum } \\
\text { and A-quorums }\end{array}$ & Randomly \\
\hline \multirow{2}{*}[26]{} & CQS-pair & $\begin{array}{l}1 / \sqrt{s} \text { or } 1 / \sqrt{m} \text {, where } s \text { is the first } \\
\text { node system size and } m \text { is the } \\
\text { second node system size }\end{array}$ & $\begin{array}{l}\text { Two nodes with different } \\
\text { cycle lengths } s<m\end{array}$ & At least 1 in $m$ slots & Randomly \\
\hline & GQS-pair & $\begin{array}{l}2 \sqrt{s}-1 / s \text { or } 2 \sqrt{m}-1 / m \text {, where } s \\
\text { is the first node system size and } m \\
\text { is the second node system size }\end{array}$ & $\begin{array}{l}\text { Two nodes with different } \\
\text { cycle lengths } s<m\end{array}$ & At least 2 in $m$ slots & Randomly \\
\hline $\begin{array}{l}\text { QueenMAC } \\
{[27]}\end{array}$ & $\begin{array}{l}\text { Dygrid }(\mathrm{H}- \\
\text { quorum and } \mathrm{V}- \\
\text { quorum) }\end{array}$ & $\begin{array}{l}k / \sqrt{s} \text {, where } k \text { can be different for } \\
\text { each node and } s \text { is the system size }\end{array}$ & Same for all nodes & $\begin{array}{c}k 1 \times k 2 \text { between } \mathrm{H}- \\
\text { quorum and } \mathrm{V}- \\
\text { quorum }\end{array}$ & Randomly \\
\hline AAA [28] & $\begin{array}{l}\text { ACQ (A-quorum } \\
\text { and S-quorum) }\end{array}$ & $\begin{array}{l}1 / \sqrt{n} \text { for cluster member and } k / \sqrt{n} \\
\text { for clusterhead, where } l \leq k \leq n\end{array}$ & Same for all nodes & $\begin{array}{l}\text { At least } 1 \text { among S- } \\
\text { quorum and at least } \\
1 \text { between S-quorum } \\
\text { and A-quorums }\end{array}$ & Randomly \\
\hline
\end{tabular}


TABLE 2: Continued.

\begin{tabular}{|c|c|c|c|c|c|}
\hline Protocol & Quorum system & Active ratio & System size (cycle length) & $\begin{array}{c}\text { Guaranteed } \\
\text { intersection size }\end{array}$ & $\begin{array}{l}\text { Quorum slot } \\
\text { assignment }\end{array}$ \\
\hline [29] & $\begin{array}{l}\text { AMQ (A- } \\
\text { quorum and S- } \\
\text { quorum) }\end{array}$ & $\begin{array}{l}1 / \sqrt{s} \text { for cluster member and } \\
s / \sqrt{m} \text { for clusterhead, where } s \text { is } \\
\text { the member system size and } m \text { is } \\
\text { the clusterhead system size. }\end{array}$ & $\begin{array}{l}\text { Two different sizes based } \\
\text { on node role (member or } \\
\text { clusterhead), } s \text { and } m\end{array}$ & $\begin{array}{l}\text { At least } 1 \text { among S- } \\
\text { quorum and at least } \\
1 \text { between S-quorum } \\
\text { and A-quorums }\end{array}$ & Randomly \\
\hline QMAC [30] & $\begin{array}{l}\text { Grid with } \\
\text { adaptive size } \\
\text { based on corona } \\
\text { traffic load }\end{array}$ & $\begin{array}{c}2 \sqrt{n}-1 / n, \text { where } n \text { is different for } \\
\text { each corona }\end{array}$ & $\begin{array}{c}\text { Based on corona traffic } \\
\text { load }\end{array}$ & At least 2 & Randomly \\
\hline $\begin{array}{l}\text { QueenMAC } \\
{[31]}\end{array}$ & SO-grid & $\begin{array}{c}m \sqrt{n} / \sqrt{n}-x \times \sqrt{n}-y, \text { where } \\
1 \leq m \leq(\sqrt{n}-x)^{2} \text { and } \\
1 \leq x=y \leq \sqrt{n} / 2\end{array}$ & $\begin{array}{l}\text { Same for all nodes } \\
\sqrt{n}-x \times \sqrt{n}-y\end{array}$ & At least $m_{1} \times m_{2}$ & Randomly \\
\hline ESQ [7] & ST-grid & $\begin{array}{l}m \sqrt{n} / w \times \sqrt{n} \text { for S-quorum sets, } \\
\text { where } 1 \leq m \leq \sqrt{n} m \times w / w \times \sqrt{n} \\
\text { for } T \text {-quorum sets, where } \\
1 \leq m \leq w\end{array}$ & Same for all nodes $w \times \sqrt{n}$ & At least $m_{1} \times m_{2}$ & Randomly \\
\hline MCQS [32] & MCQS & $\begin{array}{c}\text { Each class has a different activity } \\
\text { ratio }\end{array}$ & Same for all classes & $\begin{array}{c}\text { Can select any } \\
\text { number of } \\
\text { intersections } \\
\text { (quorums will be } \\
\text { built depending on } \\
\text { these value) }\end{array}$ & $\begin{array}{l}\text { Randomly from } \\
\text { the chosen } \\
\text { quorum class }\end{array}$ \\
\hline
\end{tabular}

(ii) System size: it is the size of the universal set from which the quorum is constructed, which is equal to the cycle length.

(iii) Guaranteed intersection size between any two quorums: an important issue to discuss in the quorum-based protocols is the guaranteed intersection slots. The efficient protocol must guarantee that there are enough intersecting slots between neighbor nodes for the data transmission. At the same time, no intersection is recommended between two hops away nodes to avoid collision. Most of the quorum-based protocols guarantee at least one or two intersections between neighbor nodes. Protocols such as that in [15], QueenMAC [27], and QTAC [31] increase the number of intersections according to nodes' traffic load.

(iv) Quorum slot assignment: the majority of the discussed protocols are assigning the quorum slots randomly to nodes. Random assignment does not always guarantee the requirements according to which the quorum system was built. Only in LASP [12], the quorums are assigned based on the node location.

In Table 3, we summarize the research works that were discussed earlier. It is important to note that these protocols did not use the same simulation parameters. From Table 3, asynchronous protocols eliminate synchronization overhead. However, nodes in these protocols need to remain awake to ensure the slot intersection for data communication. On the other hand, synchronous protocols have synchronization overhead but save more energy. Moreover, adaptive protocols that allow nodes to adapt their quorum sets according to their traffic load or delay show better performance. Finally, it is absolutely true that overhearing and idle listening can be avoided if suitable quorum sets are built, but note that using the quorum concept may increase the end-to-end delay, and therefore the network throughput may degrade.

We can conclude that, first, homogeneous protocols are more suitable with regular topologies (e.g., grid, hexagonal, and so on) where nodes have a fixed number of neighbors and similar requirements and constraints (traffic load, delay, energy, and so on). Thus, assigning quorums that have the same characteristics to nodes is more appropriate in this case. For example, nodes in LAPS [12] are placed in hexagon cells and assigned quorums with six elements, one intersection with each neighbor. In such a network, only neighbor nodes need to communicate, and thus this quorum system is perfect for satisfying this requirement. Second, if node situations (e.g., traffic load and delay requirements) can change over time, designing an adaptive quorum system is necessary to allow nodes to adapt to the new conditions easily. Differently, networks with nodes having different roles and responsibilities (e.g., clustered networks and vehicular networks) need quorums that satisfy these different roles, and hence asymmetric protocol can be designed for such networks. For example, for the vehicular networks, nodes have different roles (e.g., vehicles, roadside units, and so on), and they also need to adjust their quorums due to the vehicles' high mobility and the delay requirement. Therefore, it is more suitable for such a network to build an adaptive asymmetric quorum system.

An important finding from this survey is that many quorum-based sleep/wakeup scheduling MAC protocols are designed without considering the end-to-end delay. In most research studies, a limited number of intersections are used to save nodes' energy. Indeed, if a node receives a data 
TABLE 3: Summary of quorum-based sleep/wakeup scheduling MAC protocols.

\begin{tabular}{|c|c|c|c|c|c|}
\hline Protocol & & Objective & Synchronization & Performance & Limitation \\
\hline $\begin{array}{l}\text { Homogeneous with } \\
\text { fixed intersection } \\
\text { size }\end{array}$ & LAPS [12] & $\begin{array}{l}\text { Reduces energy } \\
\text { consumption and end-to- } \\
\text { end delay by allowing only } \\
\text { the neighbor cell to have a } \\
\text { common wakeup slot. }\end{array}$ & Required & $\begin{array}{l}\text { (i) Shows better } \\
\text { performance in terms of } \\
\text { energy efficiency and } \\
\text { packet loss ratio as } \\
\text { compared to IEEE } \\
802.11 \text { PSM and AQBC. } \\
\text { (ii) Reduces end-to-end } \\
\text { delay with high-density } \\
\text { networks when } \\
\text { compared to IEEE } \\
\text { 802.11 PSM and AQBC. }\end{array}$ & $\begin{array}{l}\text { (i) Collision when } \\
\text { more than one node } \\
\text { may exist in the } \\
\text { same cell. } \\
\text { (ii) High end-to-end } \\
\text { delay with low- } \\
\text { density networks. }\end{array}$ \\
\hline \multirow[b]{3}{*}{$\begin{array}{l}\text { Homogeneous with } \\
\text { limited intersection } \\
\text { size }\end{array}$} & $\begin{array}{c}\text { QMMAC } \\
{[13]}\end{array}$ & $\begin{array}{l}\text { Takes advantage of both } \\
\text { the quorum concept and } \\
\text { the multichannel } \\
\text { communication feature to } \\
\text { highly increase the } \\
\text { throughput while saving } \\
\text { energy. }\end{array}$ & Required & $\begin{array}{l}\text { Shows better } \\
\text { performance in terms of } \\
\text { end-to-end delay and } \\
\text { energy efficiency as } \\
\text { compared to grid } \\
\text { quorum system. }\end{array}$ & $\begin{array}{l}\text { (i) Collision when } \\
\text { two neighbors are } \\
\text { assigned the same } \\
\text { channel set. }\end{array}$ \\
\hline & S-grid [14] & $\begin{array}{l}\text { Provides an alternative to } \\
\text { the grid quorum system } \\
\text { with better neighborhood } \\
\text { sensitivity. }\end{array}$ & Not required & $\begin{array}{l}\text { Shows better neighbor } \\
\text { sensibility and } \\
\text { transmission delay } \\
\text { between nodes as } \\
\text { compared to the grid } \\
\text { quorum system. }\end{array}$ & $\begin{array}{l}\text { (i) Control packet } \\
\text { collision. } \\
\text { (ii) High end-to-end } \\
\text { delay. }\end{array}$ \\
\hline & [15] & $\begin{array}{l}\text { Provides continuous } \\
\text { intersections between } \\
\text { quorums to transmit burst } \\
\text { traffics. }\end{array}$ & Not required & $\begin{array}{l}\text { (i) Reduces end-to-end } \\
\text { delay by } 30 \% \text { and } \\
\text { increases throughput } \\
\text { and energy conservation } \\
\text { by } 40 \% \text { as compared to } \\
\text { the non-continuous } \\
\text { quorum-based unicast } \\
\text { protocols (grid and } \\
\text { torus). } \\
\text { (ii) Reduces end-to-end } \\
\text { delay by } 35 \% \text { and } \\
\text { increases throughput } \\
\text { and energy conservation } \\
\text { by } 20 \% \text { as compared to } \\
\text { the non-continuous } \\
\text { quorum-based multicast } \\
\text { protocols. }\end{array}$ & $\begin{array}{l}\text { (i) Exposed terminal } \\
\text { problem. } \\
\text { (ii) Control packet } \\
\text { collision. }\end{array}$ \\
\hline
\end{tabular}


TABle 3: Continued.

\begin{tabular}{|c|c|c|c|c|c|}
\hline Protocol & & Objective & Synchronization & Performance & Limitation \\
\hline \multirow{7}{*}{$\begin{array}{l}\text { Adaptive } \\
\text { heterogeneous with } \\
\text { limited intersection } \\
\text { size }\end{array}$} & AQEC [16] & $\begin{array}{l}\text { Allows nodes to adjust } \\
\text { their grid quorum based on } \\
\text { their load. }\end{array}$ & Not required & $\begin{array}{l}\text { Shows higher delay } \\
\text { compared to IEEE } \\
\text { 802.11 PSM while } \\
\text { achieving higher energy } \\
\text { conservation. }\end{array}$ & $\begin{array}{l}\text { (i) Control packet } \\
\text { collision. } \\
\text { (ii) High end-to-end } \\
\text { delay. }\end{array}$ \\
\hline & $\begin{array}{l}\text { HQPS } \\
{[17,18]}\end{array}$ & $\begin{array}{l}\text { Allows nodes with different } \\
\text { requirements to have } \\
\text { different cycle lengths } \\
\text { while guaranteeing the } \\
\text { intersection between any } \\
\text { two nodes. }\end{array}$ & $\begin{array}{l}\text { (i) Synchronization } \\
\text { required in [17]. } \\
\text { (ii) Work in [18] has } \\
\text { two cycle patterns for } \\
\text { synchronous and } \\
\text { asynchronous nodes. }\end{array}$ & $\begin{array}{c}\text { Increases energy } \\
\text { conservation by } 31 \% \text { as } \\
\text { compared to AQEC. }\end{array}$ & $\begin{array}{l}\text { (i) Control packet } \\
\text { collision. } \\
\text { (ii) High end-to-end } \\
\text { delay. }\end{array}$ \\
\hline & As-grid [19] & $\begin{array}{l}\text { Provides adaptive S-grid to } \\
\text { allow nodes to have } \\
\text { different cycle lengths. }\end{array}$ & Not required & $\begin{array}{l}\text { Shows better neighbor } \\
\text { sensibility as compared } \\
\text { to the grid and S-grid } \\
\text { quorum systems. }\end{array}$ & $\begin{array}{l}\text { (i) Control packet } \\
\text { collision. } \\
\text { (ii) High end-to-end } \\
\text { delay. }\end{array}$ \\
\hline & TLS $[20,21]$ & $\begin{array}{l}\text { Allows nodes to adjust } \\
\text { their grid quorum based on } \\
\text { their load. }\end{array}$ & Required & $\begin{array}{c}\text { Performs better in terms } \\
\text { of energy efficiency as } \\
\text { compared to QMAC and } \\
\text { AQBC. }\end{array}$ & $\begin{array}{l}\text { (i) Collision (same } \\
\text { forwarder set). } \\
\text { (ii) Increases end- } \\
\text { to-end delay. }\end{array}$ \\
\hline & HMAC [22] & $\begin{array}{l}\text { Allows nodes to adjust } \\
\text { their grid quorum based on } \\
\text { their pending packet. }\end{array}$ & Required & $\begin{array}{l}\text { Performs better in terms } \\
\text { of energy efficiency as } \\
\text { compared to QMAC in } \\
\text { one-hop network. }\end{array}$ & $\begin{array}{l}\text { (i) Not suitable for } \\
\text { multihop network. }\end{array}$ \\
\hline & AQPS [23] & $\begin{array}{l}\text { Adjusts its grid size with } \\
\text { one condition that the } \\
\text { same column is chosen } \\
\text { every time. }\end{array}$ & Not required & $\begin{array}{c}\text { Shows better energy } \\
\text { efficiency as compared } \\
\text { to the grid quorum } \\
\text { system. }\end{array}$ & $\begin{array}{l}\text { (i) Control packet } \\
\text { collision. } \\
\text { (ii) High end-to-end } \\
\text { delay. }\end{array}$ \\
\hline & OFAA [24] & $\begin{array}{l}\text { Allows any two } \\
\text { asynchronous nodes with } \\
\text { different cycle lengths to } \\
\text { have at least one } \\
\text { intersection. }\end{array}$ & Not required & $\begin{array}{l}\text { Performs better in terms } \\
\text { of survival ratio and end- } \\
\text { to-end energy } \\
\text { throughput as compared } \\
\text { to AQBC. }\end{array}$ & $\begin{array}{l}\text { Collision when } \\
\text { nodes start sending } \\
\text { simultaneously. }\end{array}$ \\
\hline \multirow{2}{*}{$\begin{array}{l}\text { Asymmetric } \\
\text { heterogeneous with } \\
\text { limited intersection } \\
\text { size }\end{array}$} & ACQ [25] & $\begin{array}{l}\text { Allows nodes with different } \\
\text { roles to have different } \\
\text { wakeup schedules. }\end{array}$ & Not required & $\begin{array}{c}\text { Reduces around } 52 \% \\
\text { and } 36 \% \text { of energy } \\
\text { consumption compared } \\
\text { to AQCE and grid, } \\
\text { respectively. }\end{array}$ & $\begin{array}{l}\text { (i) Control packet } \\
\text { collision. } \\
\text { (ii) High end-to-end } \\
\text { delay. }\end{array}$ \\
\hline & [26] & $\begin{array}{l}\text { Allows two nodes with } \\
\text { different cycle lengths (n } \\
\text { and } m \text { where } n<m \text { ) and } \\
\text { uses two different quorums } \\
\text { as wakeup schedules. }\end{array}$ & Not required & $\begin{array}{l}\text { Performs better in terms } \\
\text { of discovery delay as } \\
\text { compared to the grid } \\
\text { quorum system. }\end{array}$ & $\begin{array}{l}\text { (i) Control packet } \\
\text { collision. } \\
\text { (ii) High end-to-end } \\
\text { delay. }\end{array}$ \\
\hline $\begin{array}{l}\text { Adaptive } \\
\text { asymmetric } \\
\text { heterogeneous with } \\
\text { fixed intersection } \\
\text { size }\end{array}$ & $\begin{array}{c}\text { QueenMAC } \\
\text { [27] }\end{array}$ & $\begin{array}{l}\text { Allows nodes to adjust its } \\
\text { grid quorum based on their } \\
\text { traffic load and reduces } \\
\text { collision by utilizing } \\
\text { multiple channels. }\end{array}$ & Required & $\begin{array}{l}\text { Shows better } \\
\text { performance in terms of } \\
\text { end-to-end delay and } \\
\text { energy efficiency as } \\
\text { compared to QMAC. }\end{array}$ & $\begin{array}{l}\text { (i) Collision (same } \\
\text { forwarder set). } \\
\text { (ii) High end-to-end } \\
\text { delay. }\end{array}$ \\
\hline
\end{tabular}


TABLE 3: Continued.

\begin{tabular}{|c|c|c|c|c|c|}
\hline Protocol & & Objective & Synchronization & Performance & Limitation \\
\hline $\begin{array}{l}\text { Adaptive } \\
\text { asymmetric } \\
\text { heterogeneous with } \\
\text { limited intersection } \\
\text { size }\end{array}$ & QTSAC [31] & $\begin{array}{l}\text { Allows nodes to adapt their } \\
\text { wakeup schedules } \\
\text { according to their delay } \\
\text { requirements. }\end{array}$ & Not required & 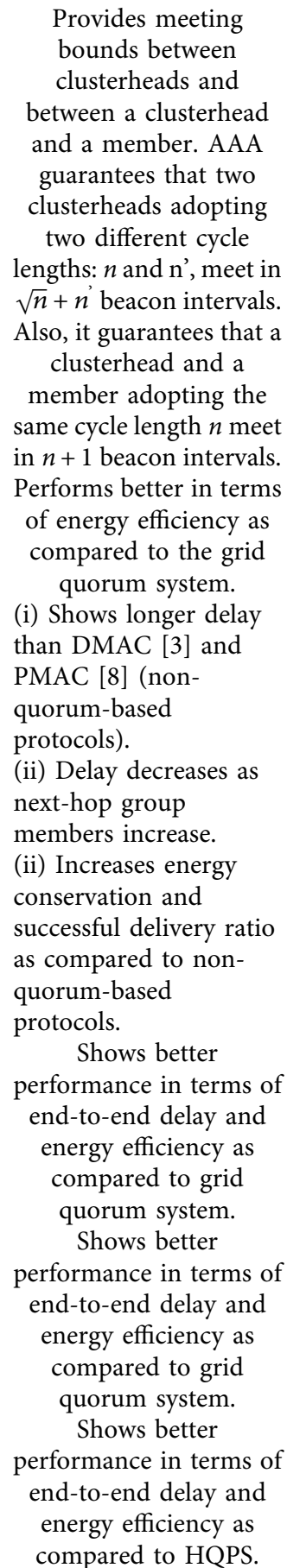 & $\begin{array}{l}\text { (i) Collision (same } \\
\text { forwarder set). } \\
\text { (ii) High end-to-end } \\
\text { delay. }\end{array}$ \\
\hline
\end{tabular}

packet, it must buffer it and wait for an intersecting wakeup slot before forwarding the buffered data to its recipient. Therefore, with fewer intersecting wakeup slots, the waiting time from node to node will increase, and hence the end-to-end delay is extremely increased due to multihop communication. The most important thing to consider is not to infinitely increase the number of intersections but to have enough intersections for nodes to transmit their traffic. Thus, as a future research direction, it is recommended to analytically derive the optimal number of intersections for each node in each communication scenario. Thus, the waiting time between nodes is reduced as well as the delay.

The self-adaptation of the schedule is another possible area of research. Nodes have to adapt their quorum sets autonomously without help from the sink or the clusterhead. Thus, the control packet overhead is decreased, and the energy efficiency is increased. 
Another important topic that has to be deeply investigated is the quorum set judicious assignment. The quorum set assignment has to be built such that neighbor nodes are assigned unique sets with unique intersections to further reduce the idle listening and the overhearing as well as to avoid or mitigate collisions. Accordingly, the assignment must be carefully designed to guarantee the needed requirements, not randomly as in the previous protocols.

There are other challenges that must be considered when designing a quorum-based scheduling protocol to optimize the protocol performance, such as finding the optimal active ratio and cycle length for each node and minimizing control packets and synchronization overhead. Considering all the above discussion, there is still a need for new quorum-based MAC protocols that strike a balance between energy efficiency and QoS, such as throughput and delay.

\section{Conclusions}

Many researchers have studied energy efficiency in energyconstrained wireless networks to extend the network lifetime. One of the most effective ways of conserving energy is the sleep/wakeup scheduling protocol, where nodes switch to sleep mode to save energy. In this paper, we reviewed a number of existing sleep/wakeup scheduling MAC protocols that utilize the quorum concept to increase energy efficiency. This survey paper is the pioneer survey that discusses several synchronous and asynchronous quorum-based sleep/wakeup scheduling MAC protocols. First, we discussed the concept of quorum systems and the use of these systems to design energy-efficient sleep/wakeup scheduling MAC protocol. Then, the quorum-based sleep/wakeup scheduling MAC protocols are classified according to their quorum system characteristics into homogeneous and heterogeneous. Moreover, these protocols are classified according to the guaranteed intersection between any two quorum sets into fixed and limited. We deeply investigated these quorum-based sleep/wakeup scheduling protocols. We compared and discussed these protocols. This helped us to define the challenges of designing such a protocol. According to our detailed comparison, we can conclude that homogeneous protocols are more suitable for nodes with similar requirements and constraints. Differently, heterogeneous protocols are more suitable when nodes' situations, roles, and responsibilities change over time. Based on this survey, we observe that many quorum-based MAC protocols are designed without taking into consideration the end-to-end delay, the self-adaptation of the quorum sets, and the proper assignment of quorum sets. To conclude, new quorum-based MAC protocols that strike a balance between energy efficiency and QoS are needed.

\section{Data Availability}

The data used to support the findings of this study are included within the article.

\section{Conflicts of Interest}

The authors declare that there are no conflicts of interest regarding the publication of this paper.

\section{References}

[1] H. H. R. Sherazi, L. A. Grieco, and G. Boggia, "A comprehensive review on energy harvesting MAC protocols in WSNs: challenges and tradeoffs," Ad Hoc Networks, vol. 71, pp. 117-134, 2018.

[2] W. Ye, J. Heidemann, and D. Estrin, "An energy-efficient MAC protocol for wireless sensor networks," IEEE Computer and Communications Societies, vol. 3, pp. 1567-2157, 2002.

[3] G. Lu, B. Krishnamachari, and C. S. Raghavendra, "An adaptive energy-efficient and low-latency MAC for tree-based data gathering in sensor networks," Wireless Communications and Mobile Computing, vol. 7, no. 7, pp. 863-875, 2007.

[4] J. Polastre, J. Hill, and D. Culler, "Versatile low power media access for wireless sensor networks," in Proceedings of the SenSys'04 -2nd International Conference on Embedded Networked Sensor Systems, pp. 95-107, New York, NY, USA, November 2004.

[5] I. Rhee, A. Warrier, M. Aia, and J. Min, "ZMAC: a hybrid MAC for wireless sensor networks," in Proceedings of the SenSys 20052nd International Conference on Embedded Networked Sensor Systems, Baltimore, MD, USA, November 2005.

[6] L. Tang, Y. Sun, O. Gurewitz, and D. B. Johnson, "PW-MAC: an energy-efficient predictive-wakeup MAC protocol for wireless sensor networks," in Proceedings of the IEEE INFOCOM, pp. 1305-1313, Shanghai, China, April 2011.

[7] Y. Liu, X. Liu, M. Zhao, K. K. L. Wong, and M. Ma, “An element shift quorum based medium access protocol for Internet of things," IEEE Transactions on Vehicular Technology, vol. 70, no. 7, pp. 7113-7126, 2021.

[8] T. Zheng, S. Radhakrishnan, and V. Sarangan, "PMAC: an adaptive energy-efficient MAC protocol for wireless sensor networks," in Proceedings of the IEEE International Parallel and Distributed Processing Symposium, Denver, CO, USA, April 2005.

[9] Y.-C. Kuo and C.-C. Hsu, "Asymmetric quorum-based power-saving protocols in asynchronous clustered ad hoc networks," Adhoc \& Sensor Wireless Networks, vol. 46, 2020.

[10] S. Sarang, G. M. Stojanović, S. Stankovski, Z. Trpovski, and M. Drieberg, "Energy-efficient asynchronous QoS MAC protocol for wireless sensor networks," Wireless Communications and Mobile Computing, vol. 2020, Article ID 8860371, 13 pages, 2020.

[11] H. Cho and J. So, "Oppo-flood: an energy-efficient data dissemination protocol for asynchronous duty-cycled wireless sensor networks," Wireless Communications and Mobile Computing, vol. 2019, Article ID 7651861, 19 pages, 2019.

[12] C.-T. Chang, C.-Y. Chang, C.-H. Kuo, and C.-Y. Hsiao, "A location-aware power saving mechanism based on quorum systems for multi-hop mobile ad hoc networks," Ad Hoc Networks, vol. 53, pp. 94-109, 2016.

[13] E. Alzahrani and F. Bouabdallah, "QMMAC: quorum-based multichannel MAC protocol for wireless sensor networks," Sensors, vol. 21, no. 11, 2021.

[14] M. Imani and M. D. T. Fooladi, "S-grid: a new quorum-based power saving protocol to maximize neighbor sensibility," in Proceedings of the 2017 Iranian Conference on Electrical Engineering (ICEE), pp. 2134-2139, Tehran, Iran, May 2017.

[15] Y.-C. Kuo and X.-W. Zhou, "Continuous quorum-based multicast power-saving protocols in the asynchronous ad hoc network for burst traffics," Ad Hoc Networks, vol. 74, pp. 1-16, 2018.

[16] C.-M. Chih-Min Chao, J.-P. Jang-Ping Sheu, and I.-C. I-Cheng Chou, "An adaptive quorum-based energy conserving protocol 
for IEEE 802.11 ad hoc networks," IEEE Transactions on Mobile Computing, vol. 5, no. 5, pp. 560-570, 2006.

[17] S. H. Wu, M. S. Chen, and C. M. Chen, "Fully adaptive power saving protocols for ad hoc networks using the Hyper Quorum System," in Proceedings of the 28th International Conference on Distributed Computing Systems ICDCS 2008, Beijing, China, June 2008.

[18] S. H. Wu, M. S. Chen, and C. M. Chen, "Optimally adaptive power-saving protocols for ad hoc networks using the hyper quorum system," IEEE/ACM Transactions on Networking, vol. 22, pp. 1-15, 2014.

[19] M. Imani, O. Noshiri, M. Joudaki, M. Pouryani, and M. Dehghan, “Adaptive S-grid: a new adaptive quorum-based power saving protocol for multi-hop ad hoc networks," in Proceedings of the 2017 IEEE 4th International Conference on Knowledge-Based Engineering and Innovation (KBEI), pp. 470-475, Tehran, Iran, December 2017.

[20] P. K. Sahoo, I. S. Hwang, and C. Y. Cheng, "An adaptive traffic load based scheduling protocol for wireless sensor networks," in Proceedings of the 2012 International Conference on Advances in Computing, Communications and Informatics, Chennai, India, August2012.

[21] P. K. Sahoo and H. K. Thakkar, "TLS: traffic load based scheduling protocol for wireless sensor networks," International Journal of Ad Hoc and Ubiquitous Computing, vol. 30, no. 3, pp. 150-160, 2019.

[22] L. Sherly Puspha Annabel and K. Murugan, “An energy efficient wakeup schedule and power management algorithm for wireless sensor networks," in Proceedings of the International Conference on Recent Trends in Information Technology, Chennai, India, April 2012.

[23] Y.-S. Chen, H.-Z. Chen, and R.-S. Cheng, "Adaptive quorumbased power-saving protocol for asynchronous wireless ad hoc networks," Wireless Personal Communications, vol. 84, no. 3, pp. 1771-1795, 2015.

[24] Z. T. Zi-Tsan Chou, Y. H. Yu-Hsiang Lin, and T. L. TsangLing Sheu, "Asynchronous power management protocols with minimum duty cycle and maximum adaptiveness for multihop ad hoc networks," IEEE Transactions on Vehicular Technology, vol. 62, no. 7, pp. 3301-3314, 2013.

[25] S.-H. Wu, C.-M. Chen, and M.-S. Chen, "An asymmetric quorum-based power saving protocol for clustered ad hoc networks," in Proceedings of the 27th International Conference on Distributed Computing Systems (ICDCS '07), Toronto, Canada, June 2007.

[26] S. Lai, B. Ravindran, and H. Cho, "Heterogenous quorumbased wake-up scheduling in wireless sensor networks," IEEE Transactions on Computers, vol. 59, no. 11, pp. 1562-1575, 2010.

[27] G. Ekbatanifard, R. Monsefi, M. H. Yaghmaee, and S. A. Hosseini, "Queen-MAC: a quorum-based energy-efficient medium access control protocol for wireless sensor networks," Computer Networks, vol. 56, no. 8, pp. 2221-2236, 2012.

[28] S. H. Wu, C. M. Chen, and M. S. Chen, “AAA: asynchronous, adaptive, and asymmetric power management for mobile ad hoc networks," in Proceedings of the IEEE 28th Conference on Computer Communications, Rio de Janeiro, Brazil, April 2009.

[29] S. H. Shan-Hung Wu, C. M. Chung-Min Chen, and M. S. Ming-Syan Chen, "An asymmetric and asynchronous energy conservation protocol for vehicular networks," IEEE Transactions on Mobile Computing, vol. 9, no. 1, pp. 98-111, 2010.
[30] C. M. Yi-Wei Lee and Y. W. Lee, "A quorum-based energysaving MAC protocol design for wireless sensor networks," IEEE Transactions on Vehicular Technology, vol. 59, no. 2, pp. 813-822, 2010.

[31] Y. Liu, K. Ota, K. Zhang et al., "QTSAC: an energy-efficient MAC protocol for delay minimization in wireless sensor networks," IEEE Access, vol. 6, pp. 8273-8291, 2018.

[32] A. H. Saligheh, V. Hakami, and M. Dehghan, "A generalized quorum system for asynchronous sleep scheduling in multihop wireless networks," Computer Communications, vol. 109, pp. 146-161, 2017.

[33] M. Imani, M. Joudaki, H. R. Arabnia, and N. Mazhari, “A survey on asynchronous quorum-based power saving protocols in multi-hop networks," JIPS, vol. 13, pp. 1436-1458, 2017.

[34] Z. J. Haas and B. Liang, "Ad hoc mobility management with uniform quorum systems," IEEE/ACM Transactions on Networking, vol. 7, no. 2, pp. 228-240, 1999.

[35] J.-R. Jiang and W.-J. Ling, "Seeker: an adaptive and scalable location service for mobile ad hoc networks," in Proceedings of the of International Computer Symposium 2006 (ICS 2006), Seoul, Korea, October 2011.

[36] D. Liu, X. Hu, and X. Jia, "Energy efficient protocols for information dissemination in wireless sensor networks," Lecture Notes in Computer Science, vol. 3842, pp. 176-185, 2006.

[37] M. Kumar, L. Schwiebert, and M. Brockmeyer, "Efficient data aggregation middleware for wireless sensor networks," in Proceedings of the 2004 IEEE International Conference on Mobile Ad-Hoc and Sensor Systems, pp. 579-581, Lauderdale, FL, USA, October 2004.

[38] R. Atreya, N. Mittal, and S. Peri, "A quorum-based group mutual exclusion algorithm for a distributed system with dynamic group set," IEEE Transactions on Parallel and Distributed Systems, vol. 18, no. 10, pp. 1345-1360, 2007.

[39] J.-R. Jiang, "The column protocol: a high availability and low message cost solution for managing replicated data," International Journal of Information Systems, vol. 20, no. 8, pp. 687-696, 1995.

[40] C.-M. Lin, G.-M. Chiu, and C.-H. Cho, "A new quorum-based scheme for managing replicated data in distributed systems," IEEE Transactions on Computers, vol. 51, no. 12, pp. 14421447, 2002.

[41] F. Bouabdallah, "Collision avoidance energy efficient multichannel MAC protocol for UnderWater acoustic sensor networks," IEEE Transactions on Mobile Computing, vol. 18, pp. 2298-2314, 2018.

[42] C.-M. Chao, Y.-Z. Wang, and M.-W. Lu, "Multiple-rendezvous multichannel MAC protocol design for underwater sensor networks," IEEE Transactions on Systems, Man, and Cybernetics: Systems, vol. 43, no. 1, pp. 128-138, 2013.

[43] S.-D. Lang and L.-J. Mao, "A torus quorum protocol for distributed mutual exclusion," in Proceedings of the 10th International Conference on Parallel and Distributed Computing and Systems, pp. 635-638, Innsbruck, Austria, February 1998.

[44] W.-S. Luk and T.-T. Wong, "Two new quorum based algorithms for distributed mutual exclusion," in Proceedings of the International Conference on Distributed Computing Systems, pp. 100-106, Baltimore, MD, USA, May 1997.

[45] M. Maekawa, "A $N$ algorithm for mutual exclusion in decentralized systems," ACM Transactions on Embedded Computing Systems, vol. 3, pp. 145-159, 1985.

[46] J.-R. Jiang, Y.-C. Tseng, C.-S. Hsu, and T.-H. Lai, "Quorumbased asynchronous power-saving protocols for IEEE 802.11 
ad hoc networks," Mobile Networks and Applications, vol. 10, no. 1/2, pp. 169-181, 2005.

[47] D. Agrawal and A. El Abbadi, "An efficient and fault-tolerant solution for distributed mutual exclusion," ACM Transactions on Computer Systems, vol. 9, no. 1, pp. 1-20, 1991.

[48] R. H. Thomas, "A Majority consensus approach to concurrency control for multiple copy databases," ACM Transactions on Database Systems, vol. 4, no. 2, pp. 180-209, 1979.

[49] "IEEE Standard for Wireless LAN Medium Access Control (MAC) and Physical Layer (PHY) specifications," IEEE Std 802.11-1997.

[50] S. Ray, D. Starobinski, and J. B. Carruthers, "Performance of wireless networks with hidden nodes: a queuing-theoretic analysis," Computer Communications, vol. 28, no. 10, pp. 1179-1192, 2005. 